\title{
Reduced phase space of heat-carrying acoustic phonons in single-crystalline InTe
}

\author{
Shantanu Misra $\odot,{ }^{1}$ Céline Barreteau $\odot,{ }^{2}$ Jean-Claude Crivello $\odot,{ }^{2}$ Valentina M. Giordano $\odot,{ }^{3}$ John-Paul Castellan, ${ }^{4,5}$ \\ Yvan Sidis ${ }^{0},{ }^{4}$ Petr Levinský, ${ }^{6}$ Jiří Hejtmánek, ${ }^{6}$ Bernard Malaman, ${ }^{1}$ Anne Dauscher $\odot,{ }^{1}$ Bertrand Lenoir, ${ }^{1}$

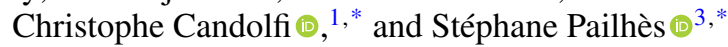 \\ ${ }^{1}$ Institut Jean Lamour, UMR 7198 CNRS - Université de Lorraine, Campus ARTEM, 2 allée André Guinier, BP 50840, 54011 Nancy, France \\ ${ }^{2}$ Univ Paris Est Creteil, CNRS, ICMPE, UMR 7182, 2 rue Henri Dunant, 94320 Thiais, France \\ ${ }^{3}$ Institute of Light and Matter, UMR 5306 Université Lyon 1-CNRS, Université de Lyon 69622 Villeurbanne Cedex, France \\ ${ }^{4}$ Université Paris-Saclay, CNRS, CEA, Laboratoire Léon Brillouin, 91191 Gif-sur-Yvette, France \\ ${ }^{5}$ Institut für Festkörperphysik, Karlsruher Institut für Technologie, D-76021 Karlsruhe, Germany \\ ${ }^{6}$ Institute of Physics, Czech Academy of Sciences, Cukrovarnická 10, 16200 Praha 6, Czech Republic
}

(Received 30 June 2020; revised 25 September 2020; accepted 12 November 2020; published 14 December 2020)

\begin{abstract}
Chalcogenide semiconductors and semimetals are a fertile class of efficient thermoelectric materials, which, in most cases, exhibit very low lattice thermal conductivity $\kappa_{\mathrm{ph}}$ despite lacking a complex crystal structure such as the tetragonal binary compound InTe. Our measurements of $\kappa_{\mathrm{ph}}(T)$ in single-crystalline InTe along the $c$ axis show that $\kappa_{\mathrm{ph}}$ exhibits a smooth temperature dependence upon cooling to about $50 \mathrm{~K}$, the temperature below which a strong rise typical for dielectric compounds is observed. Using a combination of first-principles calculations, inelastic neutron scattering (INS), and low-temperature specific heat and transport properties measurements on single-crystalline InTe, we show that the phonon spectrum exhibits well-defined acoustic modes, the energy dispersions of which are constrained to low energies due to distributions of dispersionless, optical modes, which are responsible for a broad double peak structure in the low-temperature specific heat. The latter are assigned to the dynamics of $\mathrm{In}^{+}$cations in tunnels formed by edge-sharing $\left(\mathrm{In}^{3+} \mathrm{Te}_{4}{ }^{2-}\right)^{-}$tetrahedra chains, the atomic thermal displacement parameters of which, probed as a function of temperature by means of single-crystal $\mathrm{x}$-ray diffraction, suggest the existence of a complex energy potential. Indeed, the $\mathrm{In}^{+}$-weighted optical modes are not observed by INS, which is ascribed to the anharmonic broadening of their energy profiles. While the low $\kappa_{\mathrm{ph}}$ value of $1.2 \mathrm{~W} \mathrm{~m}^{-1} \mathrm{~K}^{-1}$ at $300 \mathrm{~K}$ originates from the limited energy range available for acoustic phonons, we show that the underlying mechanism is specific to InTe and argue that it is likely related to the presence of local disorder induced by the $\mathrm{In}^{+}$site occupancy.
\end{abstract}

DOI: 10.1103/PhysRevResearch.2.043371

\section{INTRODUCTION}

The chemical engineering of the unit cell of complex crystals at the nanometer scale has emerged as one of the main cost-effective approaches for optimizing material performances in energy conversion devices such as those based on thermoelectric (TE) or photovoltaic effects [1-3]. For instance, it allows the disentanglement of the electronic from the thermal transport in semiconductors, which is used to design new efficient TE materials by lowering their lattice thermal conductivity $\kappa_{\text {ph }}$ while preserving a high and tunable electrical conductivity $[1,2]$. Although the structural complexity is known as a powerful approach for decreasing significantly $\kappa_{\mathrm{ph}}$ in crystals, the underlying microscopic mechanisms are

\footnotetext{
*Corresponding authors: christophe.candolfi@univ-lorraine.fr; stephane.pailhes@univ-lyon1.fr

Published by the American Physical Society under the terms of the Creative Commons Attribution 4.0 International license. Further distribution of this work must maintain attribution to the author(s) and the published article's title, journal citation, and DOI.
}

not yet fully understood, preventing any further theoretical prediction or screening of highly efficient TE materials $[4,5]$.

Chalcogenide semiconductors and semimetals are an important class of highly efficient TE materials, the thermal transport properties of which conform to this requirement $[1,2,6,7]$. Lying at the borders between covalent solids and classical metals, these compounds, recently dubbed as incipient metals [8], are often found to be close to a lattice instability from which strong lattice anharmonicity emerges [9]. This general mechanism, exemplified in the TE chalcogenides $\mathrm{PbTe}$ and $\mathrm{SnSe}$ [9-12], is the key characteristic explaining the very low lattice thermal conductivity $\kappa_{\mathrm{ph}}$ achieved at high temperatures despite the relative simplicity of their crystal structure. Combined with high thermopower $\alpha$ and low electrical resistivity $\rho$ when appropriately doped, it is this remarkable trait that leads to the high dimensionless TE figure of merit $Z T=\alpha^{2} T / \rho\left(\kappa_{\mathrm{ph}}+\kappa_{e}\right)$, where $\kappa_{e}$ is the electronic thermal conductivity $[1,2,6,7]$.

As another member of this technologically relevant broad family of compounds, the binary InTe has recently emerged as an interesting TE material, the performances of which rival those achieved in $\mathrm{PbTe}$, with a maximum $\mathrm{ZT}$ of 0.9 achieved at $600 \mathrm{~K}$ in pristine polycrystalline samples [13-16]. 
(a)

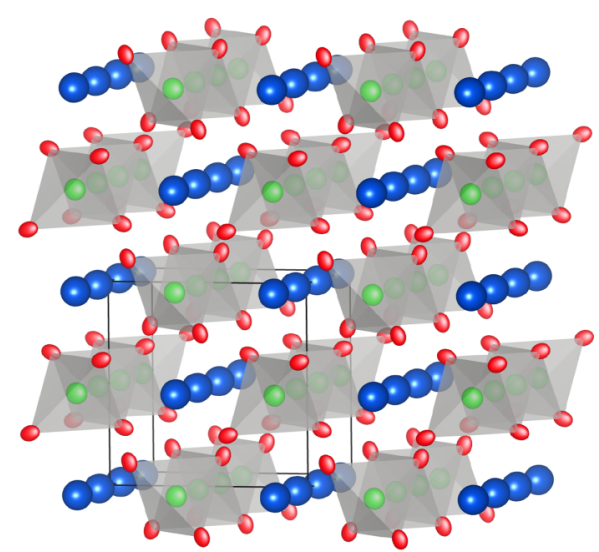

(b)

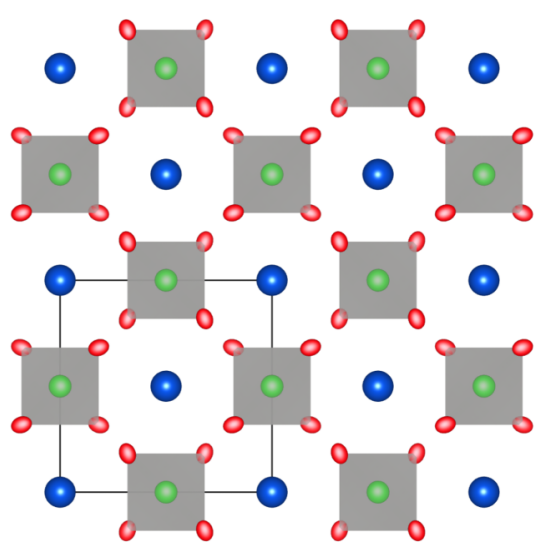

FIG. 1. (a) Perspective view of the crystal structure of InTe. In1 $(4 a)$, In $2(4 b)$, and Te $(8 h)$ atoms in the space group $14 / \mathrm{mcm}$ are shown in blue, green, and red, respectively. The In1 atoms reside in large tunnels created by the octahedra, forming infinitelike, onedimensional chains. The thermal ellipsoids are drawn at the $97 \%$ probability level. (b) Crystal structure of InTe projected along the $c$ direction.

InTe crystallizes in a TlSe structure type described in the tetragonal space group $I 4 / \mathrm{mcm}$ (no. 140) under ambient conditions (Fig. 1) [17]. While Te atoms solely occupy one crystallographic site (8h), In atoms are distributed over two independent crystallographic sites ( $4 a$ and $4 b$ ), named hereafter In1 and In2, respectively. Due to distinct bonding environment between the two $\mathrm{In}$ atoms, both $\mathrm{In}^{+}$(In1 atoms) and $\operatorname{In}^{3+}(\operatorname{In} 2$ atoms) have been proposed to be present in equal proportion in InTe resulting in an extended chemical formula written as $\mathrm{In}^{+} \mathrm{In}^{3+} \mathrm{Te}_{2}$ [12]. The $\mathrm{In}^{3+}$ ions are tetrahedrally coordinated with $\mathrm{Te}^{2-}$ ions forming edge-sharing $\left(\mathrm{In}^{3+} \mathrm{Te}_{4}{ }^{2-}\right)^{-}$tetrahedra chains, weakly bonded to each other. These long, negatively charged atomic chains are elongated along the tetragonal $c$ direction. $\mathrm{In}^{+}$ions reside in tunnels formed by these chains and are surrounded by 8-Te atoms, forming an eightfold tetragonal antiprismatic coordination [17]. Recent low-temperature structural investigations have suggested the existence of a superstructural ordering that sets in at $175 \mathrm{~K}$ followed by a possible charge-density wave (CDW) transition upon further cooling to $T_{\mathrm{CDW}} \approx 87 \mathrm{~K}$ in Te-deficient polycrystalline samples [16]. Both structural modulations are accompanied by a lattice distortion described in the orthorhombic space groups Ibam and Pbca, respectively [16].

One of its remarkable key properties is the very low $\kappa_{\mathrm{ph}}$ reaching $\sim 0.3-0.4 \mathrm{~W} \mathrm{~m}^{-1} \mathrm{~K}^{-1}$ at $600 \mathrm{~K}$ in polycrystalline samples [13-16]. The origin of these low values, about five times lower that those measured in PbTe [1,2,6,7], has been ascribed to the loosely bound $\mathrm{In} 1$ atoms that rattle in their oversized tunnels [13]. Lattice dynamics calculations have evidenced phonon instabilities giving rise to imaginary frequencies at the Brillouin-zone (BZ) center $\Gamma$ and along the $\Gamma$ $X-M-\Gamma$ directions $[13,15,16]$. These instabilities, related to the motion of the In 1 atoms along the $c$ axis and to the rotation of the (In2) $\mathrm{Te}_{6}$ octahedra about the $c$ axis, were suppressed upon reducing the unit-cell volume, mimicking the application of an external pressure of around $3 \mathrm{GPa}$ [13]. Based on this rattling scenario, several low-energy features in the phonon spectrum were inferred from an analysis of the low-temperature specific heat [15]. In order to unveil the microscopic mechanisms governing its lattice dynamics and to determine the extent to which this rattling scenario applies to InTe, further spectroscopic tools resolving experimentally its momentum- and energy-dependent phonon spectrum are mandatory.

Here, we investigate the low-energy phonon spectrum of single-crystalline InTe by means of inelastic neutron scattering (INS) and Raman scattering experiments. These results are complemented by first-principles calculations of the phonon spectrum and electronic structure and low-temperature measurements of the thermodynamic and thermal transport properties. In contrast to $\mathrm{PbTe}$ or $\mathrm{SnSe}$ [9-12], well-defined acoustic phonons are observed without anomalies of their dispersions and energy widths in the whole Brillouin zone. The key observation is that acoustic phonons exist only in a small energy range of a few $\mathrm{meV}$, contributing to lower the lattice thermal conductivity $\kappa_{\mathrm{ph}}$ to the values measured in InTe. Interestingly, no optical-phonon branch could be observed in INS spectra. We show that the origin of the low-energy cutoff of the acoustic branches is related to two broad distributions of optical modes in the simulated density of states. The nature of their coupling with the acoustic phonons is discussed and ascribed to the incoherent dynamics of highly disordered In1 atoms, consistent with the proposed rattling scenario [13]. The presence of these distributions manifests itself on the low-temperature specific heat which features a double-peak structure, similar to those observed in strongly disordered materials such as, e.g., cubic zirconia [18-21]. These key features concur to strongly lower the lattice thermal conductivity, the room-temperature value of which is reproduced by a simple kinetic model that takes into account estimates of the sound velocity, specific heat, and phonon lifetime determined experimentally. The binary InTe exemplifies the prominent role played by localized disorder in efficiently disrupting the heat transport in well-crystallized materials.

\section{RESULTS}

\section{A. First-principles calculations}

Phonon-dispersion curves were calculated by adopting three strategies: (i) by using the experimental lattice parameters determined at $300 \mathrm{~K}$, (ii) by relaxing the crystal 


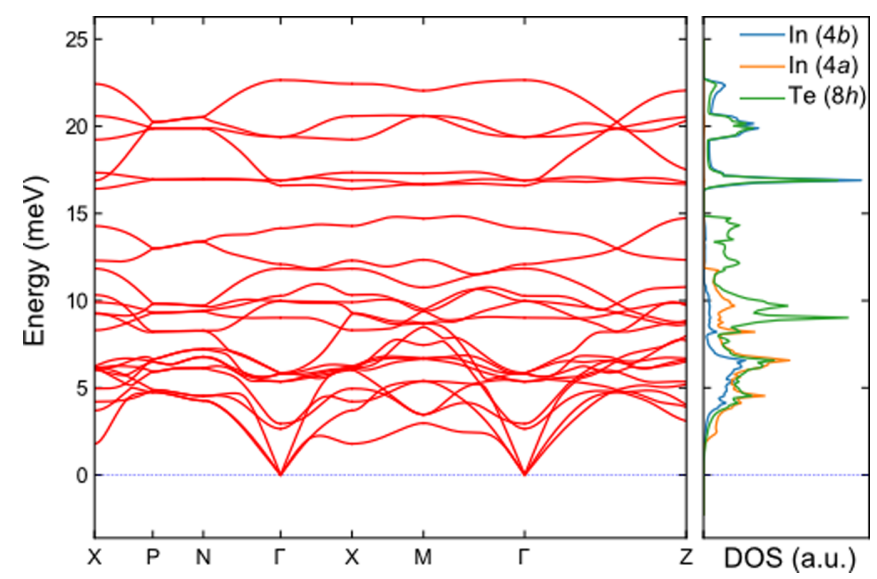

FIG. 2. Phonon-dispersion curves and projected partial phonon density of states of InTe as a function of energy calculated by considering a unit cell pressurized under $3 \mathrm{GPa}$.

structure, and (iii) by compressing the unit-cell volume up to $88 \%$ of its room-temperature value, equivalent to an external pressure of $3 \mathrm{GPa}$. The simulated phonon spectra related to the first two approaches [Fig. 8(a) in Appendix C] present some similarities with those reported by Jana et al. [13] with, most notably, the presence of imaginary frequencies of unstable observed along the $\Gamma-X-M-\Gamma$ directions, corresponding to the mechanical instabilities associated with the In1 atoms. These results confirm that the negative energies can be mostly ascribed to the dynamics of the In 1 atoms located in the large voids between the octahedra chains. Based on electron localizability indicators, these instabilities have been suggested to arise from the partially hybridized $5 s^{2}$ lone pairs of the In 1 and Te atoms [13].

$A b$ initio calculations of the free energy under pressure [Fig. 8(b) in Appendix C] show that the structure is stabilized upon compressing the unit-cell volume. The phonon spectrum obtained [Fig. 2] only exhibits positive phonon energies, confirming prior results [13]. A notable difference between our results and those reported in Ref. [13] is related to the low-energy, nearly flat optical branches along the path $\Gamma$ $X-M-\Gamma$, which shift towards higher energies in our case. Of note, considering other possible subgroups (such as $P 4_{2} / \mathrm{mcm}$ for instance), related to the $I 4 / \mathrm{mcm}$ space group reported for InTe but corresponding to a lower symmetry, did not result in the disappearance of the imaginary phonon branches. In agreement with prior results [13], the average sound velocities of the acoustic branches, extracted from the zone-center dispersions, are 2916 and $1670 \mathrm{~m} \mathrm{~s}^{-1}$ for the longitudinal and transverse polarizations, respectively. The transverse-acoustic branches merge with low-energy, flat optical branches with energies of around $\sim 2.0$ and $\sim 4.0 \mathrm{meV}$, at the $\Gamma, M$, and $X$ points. The longitudinal-acoustic branches hybridize with optical branches with energies of around $5 \mathrm{meV}$.

The projection of these dispersion curves on the energy axis yields the phonon density of states (PDOS), shown in Fig. 2. The low-energy modes are mainly associated with the atomic motion of In 1 atoms, the contribution of which mainly dominates at the lowest energies. This point is in agreement with our single-crystal $\mathrm{x}$-ray-diffraction data discussed in the next paragraph, evidencing that the In1 atoms exhibit the highest atomic thermal displacement parameters (ADPs) [13-17]. Usually, high ADPs reflect the dynamics of loosely bound atoms and are associated with low-energy, optical modes in the phonon spectrum, as observed in several cagelike materials or minerals such as tetrahedrites [22-44].

\section{B. Single-crystal $x$-ray diffraction}

The temperature dependence of the crystal structure of InTe has been investigated by means of single-crystal $\mathrm{X}$-ray diffraction (SCXRD) between 300 and $80 \mathrm{~K}$ (see Tables I to V in Appendix B for refinement details and crystallographic parameters). In agreement with prior studies [13-17], the crystal structure of InTe was successfully solved at $300 \mathrm{~K}$ in the tetragonal space group $I 4 / \mathrm{mcm}$. The $\mathrm{In} 1$ atoms, that reside in the tunnels formed by the octahedra chains, exhibit the highest ADP values while the In 2 and Te atoms show significantly lower, albeit nearly equivalent, ADP values. Upon cooling to $80 \mathrm{~K}$, the diffraction data could still be well indexed in the space group $I 4 / \mathrm{mcm}$, suggesting the absence of structural phase transition in this temperature range. This result is seemingly at odds with the tetragonal-to-orthorhombic transition observed by Back et al. [16]. near $175 \mathrm{~K}$ on powder X-ray diffraction (PXRD) data. Further checking the recorded $(h k l)$ planes in the SCXRD data, corresponding to the additional reflections appearing near $23-24^{\circ}$ in the low-temperature PXRD pattern, did not evidence the presence of additional, unindexed spots. This apparent discrepancy between both sets of data suggests a weak lattice distortion in agreement with the development of a modulated structure proposed by Back et al. [16].

The temperature dependence of the anisotropic ADP values of the In 1 atoms $\left(U_{11}=U_{22}\right.$, which corresponds to the $a b$ plane, and $U_{33}$, which corresponds to the $c$ axis, that is, along the tunnels) can be used to infer the corresponding characteristic temperatures assuming that these atoms can be described as harmonic Einstein oscillators [45]. Within this model, the temperature dependence of $U_{i i}(T)$ is given by the expression

$$
U_{i i}(T)=\frac{\hbar^{2}}{2 m k_{B} \theta_{E}} \operatorname{coth}\left(\frac{\theta_{E}}{2 T}\right)+d^{2},
$$

where $\hbar$ is the reduced Planck constant, $m$ is the reduced mass of the In atoms expressed in amu, $k_{B}$ is the Boltzmann constant, $\theta_{E}$ is the characteristic Einstein temperature of the vibration, and $d$ stands for a temperature-independent static disorder parameter. Using the $U_{i i}(T)$ values of the In1 atoms, low $\theta_{E}$ values of 26 and $35 \mathrm{~K}$ were inferred for $U_{33}$ and $U_{11}$, respectively, corresponding to characteristic energies of 2.25 and $3.0 \mathrm{meV}$, respectively. These values are higher than those determined in the cagelike compounds $\mathrm{KOs}_{2} \mathrm{O}_{6}(22 \mathrm{~K}$ or 1.9 meV [46]) and $\mathrm{VAl}_{10.1}$ compounds (down to $8 \mathrm{~K}$ or $0.7 \mathrm{meV}$ in Ga-doped $\mathrm{VAl}_{10.1}$ [31-33]) but lower than those derived in type-I clathrates (typical values between 60 and $75 \mathrm{~K}$, or between 5 and $6.5 \mathrm{meV}$ [22-30]) and for the $\mathrm{Cu} 2$ atoms (72 $\mathrm{K}$ or $6.2 \mathrm{meV}$ [34]) in tetrahedrites. These low characteristic temperatures are indicative of the presence of low-energy, dispersionless optical modes in InTe. Interestingly, the parameter $d$ exhibits a high value of $0.14 \AA$, similar to that observed 


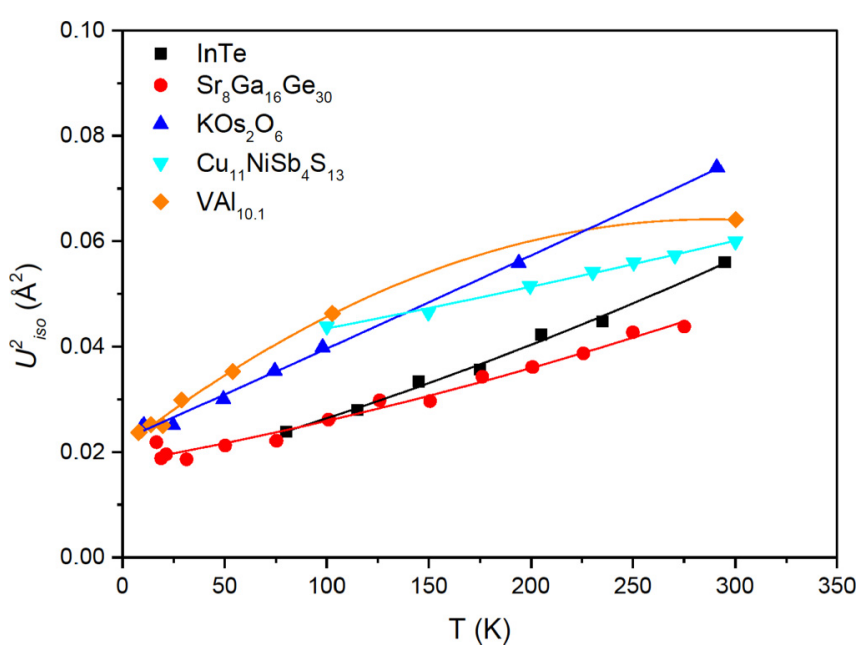

FIG. 3. Temperature dependence of the equivalent ADP values $U_{\text {iso }}$ that characterize the thermal vibrations of the In1 atoms in the tunnels. For comparison purposes, literature data obtained on the type-I clathrate $\mathrm{Sr}_{8} \mathrm{Ga}_{16} \mathrm{Ge}_{30}$, the pyrochlore $\mathrm{KOs}_{2} \mathrm{O}_{6}$, the tetrahedrite $\mathrm{Cu}_{11} \mathrm{NiSb}_{4} \mathrm{~S}_{13}$ and the cagelike compound $\mathrm{VAl}_{10.1}$ have been added (Refs. [51,47,35,32], respectively). In all these compounds, the rattling atoms ( $\mathrm{Sr}$ on the $6 d$ site of the tetrakaidecahedra cages, $\mathrm{K}, \mathrm{Cu} 2$ with a quasiplanar coordination and $\mathrm{Al}$ on the $8 a$ site of the CN16 Frank-Kaspar coordination polyhedra) have been shown to experience a complex, multiple-well potential. The solid curves are guides to the eye.

for the off-centered Ba atoms, the fundamental state of which exhibits a fourfold splitting away from the cage center, in the largest cages of several type-I clathrates [47-50]. Moreover, the isotropic $U_{\text {iso }}$ values, calculated as one-third of the trace of the orthogonalized $U_{i j}$ tensor, are similar to those measured in the above-mentioned compounds for which all the rattling atoms experience a multiple-well potential (Fig. 3). Thus, both the large $d$ and $U_{\text {iso }}$ values inferred in InTe suggest that the In1 atoms may also reside in a complex potential. This characteristic is consistent with calculations of the potential energy experienced by the In 1 atoms suggesting the presence of a double-well-shaped potential for displacements along the $c$ axis [13].

\section{Low-temperature thermodynamic and transport properties}

The low-temperature dependence of the specific heat $C_{p}$ [Fig. 9(a) in Appendix D] was analyzed according to the relation $C_{p} / T=\gamma+\beta T^{2}$ where $\gamma$ is the Sommerfeld coefficient corresponding to the electronic contribution and $\beta T^{2}$ is the phononic contribution. The very small, yet finite, $\gamma$ value of $9.74 \times 10^{-5} \mathrm{~J} \mathrm{~mol}^{-1} \mathrm{~K}^{-2}$, of the same order of magnitude as that of elemental bismuth [51], indicates a finite density of states at the Fermi level, in agreement with the semimetallic nature of InTe suggested by our electronic band structure calculations (Fig. 10 in Appendix E) and the metalliclike temperature dependence of the electrical resistivity [Fig. 9(b)] [13-17]. The $\beta$ parameter can be related to the Debye temperature $\theta_{D}$ by the relation $\theta_{D}=\left(12 \pi^{4} N R / 5 \beta\right)^{1 / 3}$ where $N$ is the number of atoms per formula unit and $R$ is the gas constant. The $\theta_{D}$ value of $169 \mathrm{~K}$ inferred is similar to those

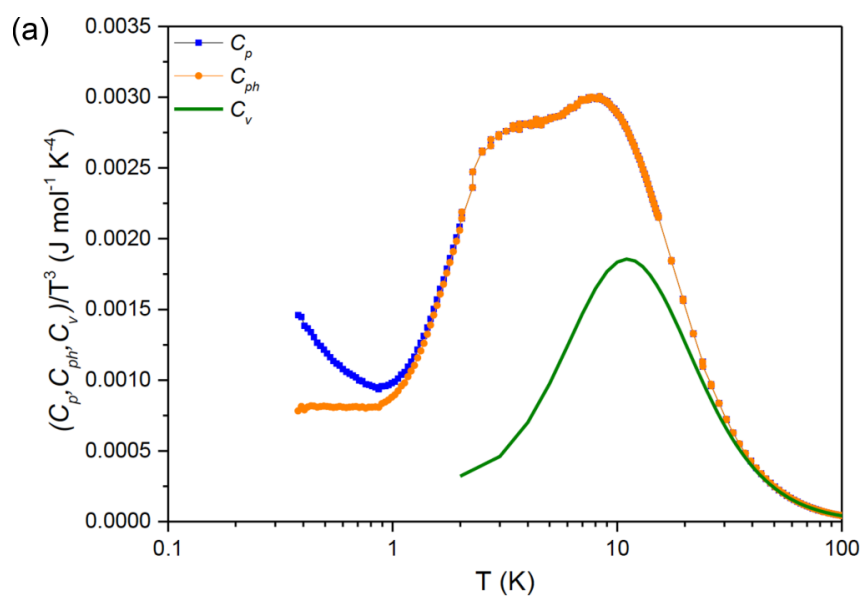

(b)

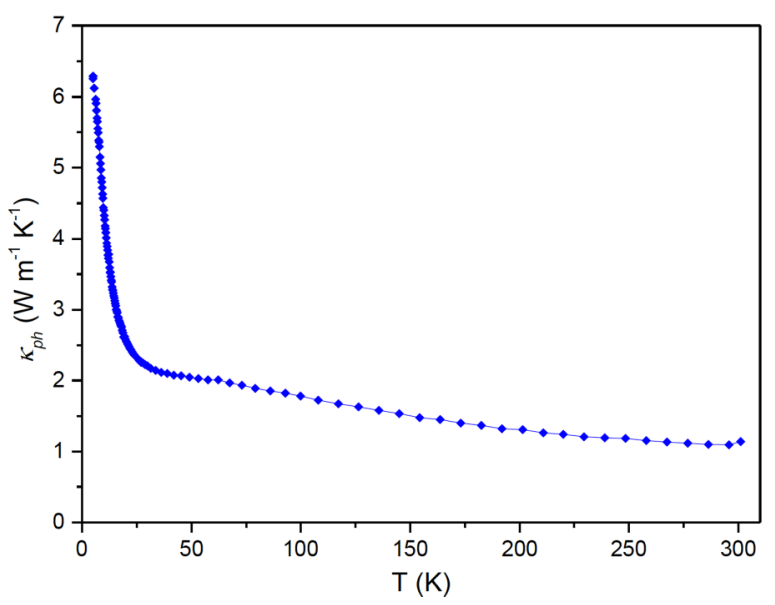

FIG. 4. (a) $C_{p} / T^{3}$ as a function of $T$ for single-crystalline InTe measured down to $0.35 \mathrm{~K}$ (blue filled squares). The electronic contribution defined as $\gamma T$, where $\gamma$ is the Sommerfeld coefficient responsible for the upturn upon cooling below $1 \mathrm{~K}$, has been subtracted to obtain the lattice contribution to the specific heat $C_{\mathrm{ph}}$ (orange filled circles). The theoretical temperature dependence of $C_{v} / T^{3}$ (green solid curve) has been derived for a unit cell pressurized under $3 \mathrm{GPa}$. (b) Temperature dependence of the lattice thermal conductivity of single-crystalline InTe measured along the $c$ axis. In both panels, the solid curves are guides to the eye.

determined in other chalcogenides such as $\operatorname{SnTe}(\sim 140 \mathrm{~K})$ or PbTe $(\sim 175 \mathrm{~K})$ and consistent with the value of $200 \mathrm{~K}$ estimated in the study of Zhu et al. [15].

The main intriguing feature that marks the $C_{p}$ of InTe as distinct among chalcogenide semiconductors is revealed by the low-temperature data plotted as $C_{p} / T^{3}$ versus $T$ [Fig. 4(a)]. This representation evidences the deviations from the Debye $T^{3}$-like temperature dependence, which accounts only for the contribution of phonons in the acoustic regime. A large excess specific heat from the Debye contribution is frequently observed in materials crystallizing with a complex unit cell [22-44]. It manifests as a single, broad hump at low temperatures, commonly attributed to low-energy, nondispersive distribution of optical modes [22-44]. The temperature at which this excess is maximum corresponds to a characteristic energy that limits the energy range of the acoustic phonons. Surprisingly, in the case of InTe, this excess is characterized by two well-defined humps, the maxima of which are located 

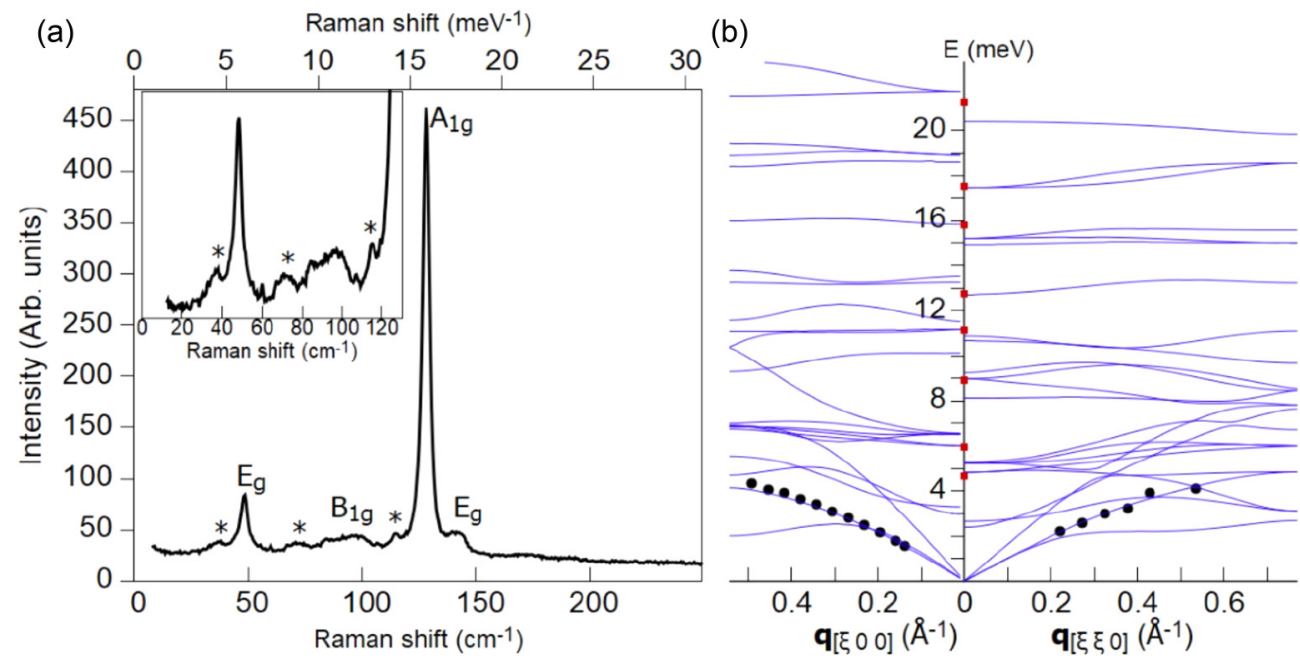

FIG. 5. (a) Low-frequency Raman spectrum of InTe measured at $300 \mathrm{~K}$. The $x$ axis is given in $\mathrm{cm}^{-1}$ (bottom) and in meV (top). The inset shows a magnification of the spectrum for frequencies lower than $130 \mathrm{~cm}^{-1}$. (b) Blue solid lines correspond to the ab initio calculated dispersions as shown in Fig. 2 along the [100] $(\Gamma-M)$ and [110] $(\Gamma-X)$ directions, respectively. The red squares at the $\Gamma$ point show the energies of the Raman peaks observed in the spectrum (a). The black filled circles give the dispersions of the transverse acoustic phonon as extracted from our INS measurements.

at 3.5 and $8 \mathrm{~K}$. The comparison of these data with $C_{v}$, inferred from the phonon spectrum calculated for a unit cell pressurized under $3 \mathrm{GPa}$ with no imaginary frequencies, shows that only the peak observed at $8 \mathrm{~K}$ is qualitatively captured while the lowest peak is not reproduced. Of relevance is the fact that a similar behavior is observed on the data collected on a polycrystalline sample (not shown), making this double-peak structure a robust property of the low-temperature specific heat of InTe.

The temperature dependence of the lattice thermal conductivity $\kappa_{\mathrm{ph}}$ measured in single-crystalline InTe along the $c$ axis is shown in Fig. 4(b). Because of the rather high $\rho$ values [Fig. 9(b)], the electronic contribution $\kappa_{e}$ is small across the entire temperature range and hence, as a first approximation, $\kappa$ is nearly equivalent to the lattice contribution $\kappa_{\text {ph }}$ [Fig. 9(c)]. The room-temperature value of $1.2 \mathrm{~W} \mathrm{~m}^{-1} \mathrm{~K}^{-1}$ is higher than that measured in polycrystalline samples $\left(0.8 \mathrm{~W} \mathrm{~m}^{-1} \mathrm{~K}^{-1}\right.$ perpendicular to the pressing direction) due to the absence of grain-boundary scattering in single crystals [13-17]. Upon cooling, $\kappa_{\mathrm{ph}}(T)$ smoothly increases up to about $2 \mathrm{~W} \mathrm{~m}^{-1} \mathrm{~K}^{-1}$ around $50 \mathrm{~K}$. At this temperature, a strong upturn in the temperature dependence is observed, with the $\kappa_{\mathrm{ph}}$ values rising rapidly down to the lowest temperature measured where $\kappa_{\mathrm{ph}} \approx 6.5 \mathrm{~W} \mathrm{~m}^{-1} \mathrm{~K}^{-1}$. The maximum, that corresponds to the dielectric maximum (often also referred to as the umklapp peak), is not yet reached at $5 \mathrm{~K}$, although the slight change in the slope observed near $5 \mathrm{~K}$ suggests that a maximum would probably be attained slightly below this temperature. The fact that the peak in $\kappa_{\mathrm{ph}}(T)$ is reached at such low temperatures, significantly lower than in polycrystalline samples $(\sim 18 \mathrm{~K})[15]$, attests to the good quality of our grown single crystal. The presence of such a well-defined peak indicates that $\kappa_{\mathrm{ph}}(T)$ in InTe retains a crystallinelike behavior, a property shared by most other chalcogenide compounds despite their inherent anharmonicity [7,9-12].

\section{Inelastic Raman and neutron scattering}

We now turn to the low-energy lattice dynamics of InTe investigated through Raman and inelastic neutron scattering (INS) measurements. From the grouptheoretical analysis, ten Raman-active modes are allowed for the Wyckoff positions $\left(8 h-\mathrm{Te}^{2-}, 4 a-\mathrm{In}^{+}\right.$, and $\left.4 b-\mathrm{In}^{3+}\right)$ with the irreducible representation $A_{1 \mathrm{~g}}(8 h)+$ $B_{1 \mathrm{~g}}(8 h)+2 B_{2 \mathrm{~g}}(8 h$ and $4 b)+3 E_{\mathrm{g}}(8 h, 4 b$, and $4 a) \quad$ [52]. An earlier polarization-dependent Raman study on InTe single crystals revealed the existence of two $E_{\mathrm{g}}$ peaks at $46 \mathrm{~cm}^{-1}$ $(5.7 \mathrm{meV})$ and $139 \mathrm{~cm}^{-1}(17.2 \mathrm{meV})$, one $A_{1 \mathrm{~g}}$ peak at 126 $\mathrm{cm}^{-1}(15.6 \mathrm{meV})$, and one $B_{1 \mathrm{~g}}$ at $86 \mathrm{~cm}^{-1}(10.7 \mathrm{meV})$ [53]. However, no additional peaks were observed below $46 \mathrm{~cm}^{-1}$. The peaks with frequencies higher than $100 \mathrm{~cm}^{-1}$ and their assignments were confirmed by a more recent combined experimental and theoretical Raman study carried out on InTe powders up to $\sim 19 \mathrm{GPa}$ [54]. The theoretical simulations show that the $A_{1 \mathrm{~g}}$ mode corresponds to motions involving only the Te atoms in the basal $a b$ plane containing the $\mathrm{In}^{3+}-\mathrm{Te}^{2-}$ tetrahedral layers. On the other hand, the $E_{\mathrm{g}}$ mode at $139 \mathrm{~cm}^{-1}$ is ascribed to the motions of the $\mathrm{In}^{3+}$ atoms in the $a b$ plane and $\mathrm{Te}^{2-}$ atoms along the $c$ direction. A representative spectrum of our Raman measurements is shown in Fig. 5(a). The $E_{\mathrm{g}}$ and $A_{1 \mathrm{~g}}$ peaks are observed with frequencies that are in agreement with those previously reported: the $E_{\mathrm{g}}$ peak at $48 \mathrm{~cm}^{-1}(5.9 \mathrm{meV})$ and $141 \mathrm{~cm}^{-1}$ and the $A_{1 \mathrm{~g}}$ peak at $128 \mathrm{~cm}^{-1}$. Additional peaks, marked by an asterisk $\left(^{*}\right)$ in the spectrum, were observed at energies below $100 \mathrm{~cm}^{-1}$, as observed in the magnification of the low-frequency range shown in the inset of Fig. 5(a). In particular, a shoulder near the $E_{\mathrm{g}}$ peak at $46 \mathrm{~cm}^{-1}$ appears at an energy of $37 \mathrm{~cm}^{-1}(4.5$ $\mathrm{meV}$ ). This shoulder can be also seen in the Raman spectrum reported in Ref. [53] using a geometry optimized for the $A_{1 \mathrm{~g}}$ symmetry. A direct comparison of our Raman frequencies with the computed phonon energies is shown in Fig. 5(b) in 

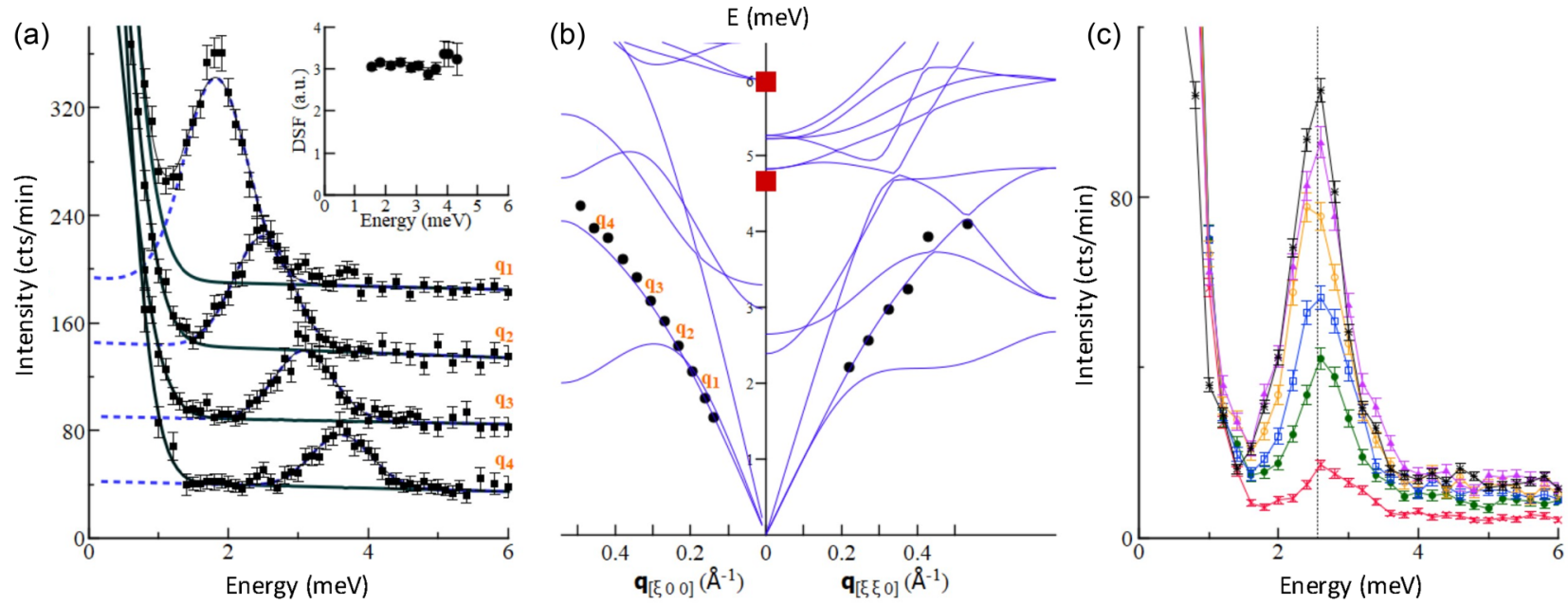

FIG. 6. (a) Inelastic neutron intensity as function of energy recorded at $300 \mathrm{~K}$ for a series of energy scans through the transverse acoustic (TA) dispersion, polarized along the [010] direction $\left(\mathrm{TA}_{100}^{010}\right.$ ), at constant wave vectors along the [100] direction labeled from $q_{1}$ to $q_{4}$. (b) Left panel: Dispersion of $\mathrm{TA}_{100}^{010}$ along [100] and polarized along [010]. The wave vectors $q_{1-4}$ are indicated. Right panel: TA dispersion along [110] and polarized along [1-10], $\mathrm{TA}_{110}^{1-10}$. Blue solid lines correspond to the ab initio calculated dispersions as shown in Fig. 2 along the [100] ( $\left.\Gamma-M\right)$ and [110] $(\Gamma-X)$ directions, respectively. The computed energies have been multiplied by a factor 1.12 for phonons propagation along $(\Gamma-M)$ and 0.9 for those propagating along $(\Gamma-X)$. The error bars of the extracted phonon energies are lower than the size of the symbols used. (c) Inelastic neutron intensity as a function of energy at a constant wave vector $Q=(6,0.2,0)\left[q_{2}\right.$ in panels (a) and (b)], which belongs to the TA dispersion shown in the left panel recorded at $300 \mathrm{~K}(*), 200 \mathrm{~K}(\boldsymbol{\Delta}), 150 \mathrm{~K}(\circ), 100 \mathrm{~K}(\square), 55 \mathrm{~K}(\bullet)$, and $4 \mathrm{~K}(\times)$. Data shown in (a) and (c) were collected on the spectrometer1T@LLB.

the full energy range of the Raman spectrum. While some of the measured peak energies are reproduced, there are still large differences between experiment and theory due to the pressurized unit cell used in our calculations.

The INS measurements were performed along two transverse acoustic (TA) phonon branches by means of INS (see experimental details in Appendix A). Representative energy cuts through the TA dispersion along the [100] direction in the conventional basis with the polarization along [010], labeled as $\mathrm{TA}_{100}^{010}$, collected around the Bragg peak (600) at $300 \mathrm{~K}$ are reported in Fig. 6(a) (see Figs. 11 and 12 in Appendix F for elastic scans and energy scans at constant wave vectors). TA phonons propagating along the direction [110] and polarized [1-10], TA 110 , were also investigated around the Bragg peak (330) (energy cuts are shown in Fig. 13 in Appendix F). Along both directions, well-defined acoustic phonons are observed in the whole BZ, the energies of which continuously disperse up to $\sim 4.1 \mathrm{meV}$ and $3.3 \mathrm{meV}$ for $\mathrm{TA}_{100}^{010}$ and $\mathrm{TA}_{110}^{1-10}$, respectively [Fig. 6(b)]. Within the Debye regime of the acoustic dispersion close to the $\mathrm{BZ}$ center, the slope corresponds to the sound velocity, which is equal to 1630 and $1530 \mathrm{~m} \mathrm{~s}^{-1}$ for $\mathrm{TA}_{100}^{010}$ and $\mathrm{TA}_{110}^{1-10}$, respectively. The energy widths of the TA phonons, which correspond to the inverse lifetimes, can be resolved only near the border of the $\mathrm{BZ}$ and is on the order of $50 \mu \mathrm{eV}$. The experimental uncertainty in the determination of the phonon energy width does not allow the extraction of a reliable energy dependence. Interestingly, the dynamical structure factor (DSF) of TA phonons remains constant up to $4.5 \mathrm{meV}$ [see inset in Fig. 6(a)].

The measured acoustic dispersions are directly compared with those simulated by ab initio calculations performed for a unit-cell volume of $0.88 V_{0}$ [see Fig. 6(b)], where $V_{0}$ is the initial volume. A good matching between the experimental and calculated dispersions is achieved by rescaling the energy axis by a factor of 1.12 and 0.9 along the $\Gamma-M$ and $\Gamma-X$ directions, respectively. A comparison without these scaling factors is shown in Fig. 5(b). Although the lower volume of the unit cell used in the calculations explains the necessity of this scaling, a fraction of these factors may signal that the description of the atomic potentials is not entirely satisfying, as reported in other complex systems [39,55-58]. In this regard, further self-consistent phonon calculations in which anharmonicity is included may solve this problem. However, the sound velocities and the bending of the acoustic branches are nevertheless well reproduced by our calculations. On the other hand, no well-defined optical modes are observed experimentally along the [100] direction while several optical-phonon branches are observed in the calculated phonon spectrum. Near the border of the BZ, where acoustic and optical branches hybridize, no enhancement effect of the optical intensity is captured by our INS data, in contrast to what has been observed in other complex systems such as type-I clathrates [39,5558] or skutterudites [59]. Along the [110] direction, two weak peaks can be assigned to optical phonons at the zone boundary from the comparison with the calculated phonon dispersions [see left panel in Fig. 6(b)]. Their energies are close to the lowest Raman peak energy observed in our Raman measurements.

Energy scans at constant wave vector $Q=(6,0.2,0)$, which corresponds to a representative TA phonon in the middle energy range of the $\mathrm{TA}_{100}^{010}$ branch, have been collected at different temperatures between 4 and $300 \mathrm{~K}$ as shown in Fig. 4(c). Within the instrumental resolution (see Appendix A), no significant anomalous temperature effect on the TA phonon energy and width is observed. Furthermore, its maximum intensity follows the ex- 
pected temperature dependence of the detailed balance factor.

\section{DISCUSSION}

Our INS study reveals that the physical mechanism governing the thermal transport in InTe is fundamentally different compared to its cousin chalcogenides PbTe or SnSe [9-12]. In $\mathrm{PbTe}$, it results from the proximity of a ferroelectric instability which manifests as a softening of a transverse-optical branch at the BZ center [12]. The hybridization of the latter with the longitudinal-acoustic branch, which extends over a large part of the BZ, results in a concomitant softening of the acoustic dispersion and a strong broadening of the phonon energy width. A similar ferroelectriclike lattice instability has been evidenced in SnSe that also involves a soft transverse-optical mode, eventually giving rise to strong lattice anharmonicity [9,10]. In InTe, the acoustic phonons appear well defined in the whole BZ without any anomalous broadening of their energy width. The key observation is that their dispersions are abruptly interrupted at low energies, which is not concomitant to an enhancement of an optical branch intensity at higher energies. From the calculated phonon spectrum, it can be assumed that the nondispersive optical branches, which are mostly associated with the In1 dynamics, are responsible for the cutting off of the acoustic branches at 3.3 and $4.1 \mathrm{meV}$. The latter energy is close to the energy of the low $E_{\mathrm{g}}$ Raman peak, while no peaks are detected at lower energy. This is confirmed by the double-hump structure observed in the $C_{p} / T^{3}$, implying the existence of two large peaks in the vibrational density of states.

In contrast to the specific heat, which is weighed by the total number of vibrational modes and thus is dominated by the optical part of the phonon spectrum, $\kappa_{\mathrm{ph}}$ is mostly dominated by the most dispersive and well-defined states, i.e., the acoustic phonons [39,55-58]. As they are limited to an energy much lower than the thermal energy at $300 \mathrm{~K}$ $(\sim 25 \mathrm{meV})$, all acoustic branches are fully occupied. Thus, their contribution to $\kappa_{\mathrm{ph}}$ can be roughly estimated by using the simplest form of the Boltzmann transport equation for phonons. Assuming three isotropic acoustic branches, their contribution to $\kappa_{\mathrm{ph}}$ reads as $\kappa_{\mathrm{ac}}=\frac{1}{3} C_{D}^{D L} v_{D} l=\frac{1}{3} C_{D}^{D L} v_{D}^{2} \frac{1}{\Gamma_{\mathrm{HWH}}}$ where $C_{D}^{D L}=3 R$ is the Dulong-Petit limit $(R$ is the ideal gas constant) and HWHM is half width at half maximum. Using an average sound velocity $v_{D}=1580 \mathrm{~m} \mathrm{~s}^{-1}$ and $\Gamma_{\mathrm{FWHM}}=$ $50 \mu \mathrm{eV}$ inferred from the fits, this simple relation yields $\kappa_{\mathrm{ac}}=$ $1.7 \mathrm{~W} \mathrm{~m}^{-1} \mathrm{~K}^{-1}$, which is close to the room-temperature value of $1.2 \mathrm{~W} \mathrm{~m}^{-1} \mathrm{~K}^{-1}$ measured experimentally. Regardless of its exact origin, the restriction of the acoustic branches at low energy contributes to lower $\kappa_{\text {ph }}$ to the measured values. On the other hand, the presence of bands of optical branches with almost no dispersion, which implies a high density of states, is known to provide a large momentum- and energy-conserving phase space for three-phonon scattering processes involving the acoustic modes, as demonstrated in type-I clathrates and several Tl-containing compounds $[39,55-58,60,61]$. The calculation of the cubic anharmonicity including the phonon energy renormalization due the quartic anharmonic terms is necessary to determine properly $\kappa_{\mathrm{ph}}(T)[62,63]$.
It is interesting to discuss the origin of the optical-acoustic hybridization in InTe in comparison with other known mechanisms observed in compounds crystallizing with a complex crystal structure that gives rise to low $\kappa_{\mathrm{ph}}$. The fact that the low-lying optical branches could not be detected by INS is related to either the weakness of their coherent dynamical structure factor, which is the case of pure Einstein or rattling vibrations (localized and uncoupled harmonic oscillators), an inherent strong disorder, or a combination of these two effects. The global phonon spectrum of InTe mirrors those observed in strongly disordered compounds such as cubic zirconia $\mathrm{ZrO}_{2}$, in which the optical modes are hardly observed by INS, which is assumed to result from the broadening of their energies due to static disorder $[18,19,64,65]$. On the other hand, the microscopic mechanism in InTe presents some similarities with that responsible for the low $\kappa_{\mathrm{ph}}$ values in complex materials such as type-I clathrates [39,55-58]. The heat conduction in these cagelike compounds is also conveyed by well-defined acoustic phonons, which exist only in a limited energy range, bordered by a continuum of nondispersive, optical-phonon branches mostly related to the dynamics of loosely bound cations entrapped in the covalent $\mathrm{Ge}$ or Si cages [39,55-58]. In the region of the $\mathrm{BZ}$ where the optical-acoustic phonons hybridize, the propagative nature of the acoustic modes disappears as they disperse towards the border of the BZ. Experimentally, this mechanism manifests as an abrupt decrease in their dynamical structure factor while, theoretically, it results in a very low phonon participation ratio $(<\sim 0.1)$, reflecting the fact that, contrary to an acoustic mode where all atoms move coherently, the acoustic-optical hybrid modes involve only a small fraction of atoms within the unit cell [39,55-58]. This has been interpreted as a signature of either phonon localization [66-68] or phonon confinement [39,55] and is responsible for a transition from propagative to diffusive heat transport. Of note, the acoustic-optical hybrid modes in type-I clathrates are coherent and are seen as well-defined peaks by INS with a clear coherent dynamical structure factor. In InTe, a striking difference is that the acoustic dynamical structure factor remains constant in the whole BZ [see inset of Fig. 4(a)] and no effect associated with an acoustic-to-optical spectral weight transfer is observed so that acoustic phonons remain highly propagative. Acoustic phonons with similar highly propagative character have been recently reported by INS in the complex crystalline phase of the quasicrystal approximant $o-\mathrm{Al}_{13} \mathrm{Co}_{4}$ [69]. The high-resolution neutron measurements performed in this study revealed that the acoustic-phonon lifetime is temperature independent and dominated by defect scattering, which has been reproduced by molecular dynamics performed on a slightly disordered supercell including a few percent of $\mathrm{Al}$ vacancies. This defect-containing model could also reproduce the low value as well as the smooth temperature dependence of $\kappa_{\mathrm{ph}}(T)$. Thus, the low $\kappa_{\mathrm{ph}}$ in $o-\mathrm{Al}_{13} \mathrm{Co}_{4}$ has been interpreted in terms of a combined effect of the structural complexity and the existence of a minute concentration of defects. In InTe, the lack of optical modes in the INS spectra might be a signature of a local disorder, which would indeed lead to a broadening of the optical branches, reducing their structure factor so that they are too weak to be observed by inelastic neutron spectroscopy. However, these modes clearly affect the low-temperature specific heat. 
This disordered nature of InTe captured by our INS measurements naturally raises the question of its structural origin. A first possibility is related to the In1 atoms residing in the tunnels that may not be strictly located on their site but slightly away, similar to the off-centering of the $\mathrm{Eu}, \mathrm{Sr}$, or Ba cations in the tetrakaidecahedra in the type-I clathrates $(\mathrm{Eu}, \mathrm{Sr}, \mathrm{Ba})_{8} \mathrm{Ga}_{16} \mathrm{Ge}_{30}[22,26,48-50,70,71]$. Both recent calculations [13] and our single-crystal $x$-ray-diffraction data are consistent with a double-well-shaped potential. This crystallographic characteristic, reminiscent of that observed in tetrahedrites [72,73], may explain the similarities of the lattice dynamics between InTe and these type-I clathrates for which the incoherent occupancy of the off-centering levels experienced by the encaged cations is responsible for a local disorder, i.e., a distribution of elastic constants, that broadens the acoustic branches [71,74]. Although refinements of our single-crystal x-ray-diffraction data considering a split of the In 1 site were unsuccessful, additional diffraction experiments probing local disorder would be worthwhile to further explore this hypothesis. Another possibility may be related to the presence of defects in InTe, a characteristic common to most of thermoelectric chalcogenides $[1,2,6,7]$. Previous studies have highlighted the possible presence of either In or Te vacancies [13-17], which may induce randomly distributed local static deformations in the environment of the In atoms and of the octahedra chains. The low value of the Sommerfeld coefficient measured in our single crystal shows that the Fermi level crosses the top of the valence bands, which may be ascribed to the presence of a small concentration of defects. Assuming that In vacancies are the main defects in InTe, the hole concentration measured by Hall effect in our single crystal $\left(7.5 \times 10^{19} \mathrm{~cm}^{-3}\right.$ at $\left.300 \mathrm{~K}\right)$ would correspond to a vacancy concentration on the order of only $0.2 \%$, a value similar to that observed in the binary $\mathrm{SnTe}$, for which $\mathrm{Sn}$ vacancies are always present $[75,76]$, with their concentration depending on the synthesis conditions used [77]. Such defects should give rise to a typical $q^{4}$ dependence of the acoustic-phonon energy width, which has not been resolved in the present experiments because of the limitation of the inelastic neutron and $\mathrm{x}$-ray techniques for measurements of the intrinsic phonon energy width in the whole BZ, i.e., the phonon lifetime [78]. Further neutron resonant spin-echo measurements will be required to determine the $q$ dependence of the acoustic-phonon lifetime.

Regardless of the exact origin of this disordered character, the induced broadening of the optical modes does affect the low-temperature thermodynamic properties, as shown by the peculiar two-peak-structure characterizing the $C_{p} / T^{3}$ data. In a prior study [15], $C_{p}(T)$ has been analyzed using a combination of Debye-like and Einstein-like contributions, with the latter describing the low-energy features in the PDOS evidenced by lattice-dynamics calculations. From the phonon spectrum, $C_{p}(T)$ may be simply decomposed into a sum of a Debye component accounting for three acoustic modes and two Einstein contributions $C_{E 1,2}$ so that $C_{p}=C_{D}+C_{E 1}+$ $C_{E 2}$, with $C_{E 1,2}$ qualitatively accounting for the two broad humps. The nature of these contributions cannot be strictly associated to well-defined, low-lying, and nondispersive optical modes as in cagelike materials or in some thermoelectric minerals (Refs. [22-38]) due to their absence in our INS data. Nevertheless, one can assume the existence of two broad dis- tributions of nondispersive vibrational modes in energy, which should be mostly localized, as the coherent INS structure factor has to be small. Within these assumptions, the $C_{p}(T)$ data were modeled by the relation

$$
\begin{aligned}
C_{p}=C_{D}+C_{E i}= & 9 N_{D} R\left(\frac{T}{\theta_{D}}\right)^{3} \int_{0}^{\theta_{D} / T} \frac{x^{4} e^{x}}{\left(e^{x}-1\right)^{2}} d x \\
& +\sum_{i} p_{i} N_{E i} R\left(\frac{\theta_{E i}}{T}\right)^{2} \frac{e^{\theta_{E i} / T}}{\left(e^{\theta_{E i} / T}-1\right)^{2}},
\end{aligned}
$$

where $C_{D}$ is the lattice contribution within the Debye model, $C_{E i}$ is the Einstein contribution of the $i$ th vibrational mode of the rattling atoms, $x=\hbar \omega / k_{B} T$ with $\hbar$ the reduced Planck constant and $k_{B}$ is the Boltzmann constant, $N_{D}$ is the number of Debye oscillators per formula unit, and $p_{i} N_{E i}$ and $\theta_{E i}$ are the spectral weight and Einstein temperature related to the $i$ th vibrational mode, respectively. Considering $\theta_{D}, N_{D}, p_{1} N_{E 1}$, $\theta_{E 1}, p_{2} N_{E 2}$, and $\theta_{E 2}$ as free parameters, the best fit to the data yields $\theta_{D}=148 \mathrm{~K}, N_{D}=1.46, p_{1} N_{E 1}=0.039, \theta_{E 1}=15.1$ $\mathrm{K}, p_{2} N_{E 2}=0.99$, and $\theta_{E 2}=45.9 \mathrm{~K}$. As shown in Fig. 7, this model does not satisfactorily capture the plateau below $1 \mathrm{~K}$ as well as the magnitude and width of the two peaks at 3.5 and $8 \mathrm{~K}$. The Einstein temperatures $\theta_{E 1}$ and $\theta_{E 2}$ obtained from the fit of the $C_{p}$ data with this simple model corresponds to energies of 1.30 and $3.95 \mathrm{meV}$, respectively. While $\theta_{E 2}$ corresponds well with the energy at which the TA dispersions are cut, further INS measurements would be needed to possibly link $\theta_{E 1}$ to the upper energy limit for acoustic phonons propagating along the $c$ axis. Finally, both Einstein temperatures differ from those inferred from the $U_{i i}(T)$ values of the In1 atoms (26 and $35 \mathrm{~K}$ for $U_{33}$ and $U_{11}$, respectively), suggesting that the harmonic approximation from which Eq. (1) is derived may not correctly describe the temperature dependence of $U_{i i}(T)$. As highlighted in the cagelike compound $\mathrm{VAl}_{10.1}$ [31-33], more complex, multiple-well potentials can also result in a quasilinear, harmoniclike temperature dependence of the $U_{i i}$ values, providing another hint that the potential

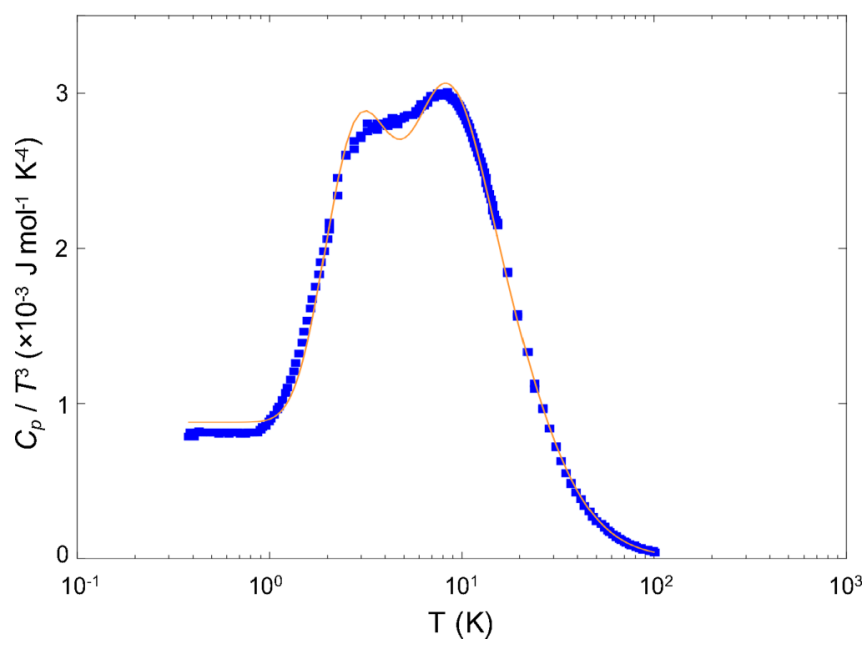

FIG. 7. Fit of the specific-heat data of single-crystalline InTe plotted as $C_{p} / T^{3}$ vs $T$ in log scale. The orange solid curve stands for the best fit to the data according to Eq. (2). 
experienced by the In1 atoms in InTe also belongs to this category. In such a case, the lowest peak in the $C_{p} / T^{3}$ data might correspond to a Schottky-like anomaly related to the dynamical disorder of the $\mathrm{In}^{+}$cations [31-33].

\section{SUMMARY AND CONCLUSION}

The low-energy phonon spectrum of InTe, probed by inelastic neutron scattering and low-frequency Raman spectroscopy on a Bridgman-grown single crystal, exhibits welldefined transverse-acoustic modes, which disperse over the whole Brillouin zone while keeping their acoustic character and their resolution-limited energy width. Their energy dispersion is constrained at 4.1 and $3.3 \mathrm{meV}$ for the modes propagating along the [100] and [110] directions, respectively. These energies correspond to the DFT calculated energies of the dispersionless bands of optical-phonon branches mostly weighted by the In 1 motions. If the cutoff at $4.1 \mathrm{meV}$ is close to the lowest peak energy observed in the Raman spectrum, no Raman signal has been detected at lower energy. While they were not detected by INS, these distributions of optical modes are found to be responsible for a broad, double-peak structure in the low-temperature specific heat. These characteristics explains the low lattice thermal conductivity values measured at $300 \mathrm{~K}$, which nevertheless retain a crystallinelike behavior at low temperatures, as shown by a well-defined dielectric maximum, likely reached slightly below $5 \mathrm{~K}$. Owing to the distinct physical picture emerging from these results, InTe clearly warrants further theoretical and experimental investigations. In particular, pinpointing the structural origin of the local disorder, which is assumed to be at the origin of the unresolved optical modes by INS, will be of interest.

\section{ACKNOWLEDGMENTS}

The authors would like to thank the Laboratoire Léon Brillouin for the support of this work and granting access to the $1 \mathrm{~T}$ inelastic neutron-scattering instruments. DFT and phonon calculations were performed using HPC resources from GENCI-CINES (Grant No. 2019-096175). S.P. and V.M.G. acknowledge the Lyon IDEX Scientific Breakthrough program for funding of the project IPPON.

\section{APPENDIX A: EXPERIMENTAL AND COMPUTATIONAL DETAILS}

A high-quality single crystal of InTe was grown by a vertical Bridgman technique using a polycrystalline ingot prepared from direct reaction of stoichiometric amounts of elemental In shots $(99.999 \%)$ and Te powders $(99.999 \%)$. To this end, both In and Te were introduced in an evacuated, sealed silica tube. The tube was placed in a rocking furnace and heated at 1023 $\mathrm{K}$, that is, above the melting point of InTe according to the In-Te binary phase diagram. This temperature was maintained during $2 \mathrm{~h}$ and was eventually quenched in room-temperature water. The obtained ingot $(15 \mathrm{~cm}$ long and $15 \mathrm{~mm}$ in diameter) was subsequently inserted into a long silica tube with a carefully designed V-shaped end to favor seed selection and growth. The sealed silica tube was then placed in a vertical two-zone furnace in which the first zone was kept constant at
$1000 \mathrm{~K}$ while the electrical power of the second zone was kept off to let the crystallization process occur at a natural cooling rate. The tube was vertically lowered through the furnace at a constant speed of $0.35 \mathrm{~mm}$ per hour with a gradient of around $15 \mathrm{~K} \mathrm{~cm}^{-1}$. After completion of the process ( 30 days), the tube was furnace-cooled to room temperature. The as-grown ingot was single crystalline from few $\mathrm{mm}$ above the tip and over a length of approximately $11 \mathrm{~cm}$.

The crystal structure and single-crystalline nature of the ingot were verified by a combination of powder $\mathrm{x}$-ray diffraction (PXRD), single-crystal x-ray diffraction (SXRD), and Laue diffraction. Powder x-ray diffraction was performed at $300 \mathrm{~K}$ using a Bruker D8 Advance diffractometer with $\mathrm{Cu}-K \alpha_{1}$ radiation $(\lambda=1.54056 \AA)$. Single-crystal $x$-ray diffraction was carried out at 300 and $80 \mathrm{~K}$ using a Bruker APEX-II diffractometer equipped with a charge-coupled device detector using Mo- $K \alpha$ radiation $(\lambda=0.71073 \AA$ ). PXRD and SXRD evidenced the absence of secondary phases and the single-crystalline nature of the ingot. The structure solution was determined by direct methods and refined by full-matrix least-squares techniques with the Sir97 and SHELX-2013 software, respectively, implemented through the WINGX program package [79]. Refinements against the SXRD data using the collected at $300 \mathrm{~K}$ confirmed that InTe crystallizes with a tetragonal structure described in the space group $I 4 / \mathrm{mcm}$ with lattice parameters $a=8.4380 \AA$ and $c=7.1413 \AA$, in good agreement with the literature data [13-17]. The lowtemperature data were also successfully refined down to 80 $\mathrm{K}$ in the space group $I 4 / \mathrm{mcm}$. Attempts at considering other lower-symmetry space groups did not improve the structural model, assessed through the reliability factors. Details of the structural refinements and the refined crystallographic parameters are listed in Tables I, II, III, IV and V in Appendix B. The density of the crystal pieces was determined to be $6.23 \mathrm{~g} \mathrm{~cm}^{-3}$ indicating that the single crystal was fully dense with a relative density of over $\sim 99 \%$.

The orientation of the crystal was determined using a three-axis goniometric head by Laue diffraction. These experiments revealed the presence of several grains in the ingot from which large single-domain crystals could be selected and subsequently cut for transport properties measurements. Of note, single-crystalline pieces could be easily cleaved along the plane (110), that is, parallel to the $c$ axis of the tetragonal crystal structure.

For transport properties measurements, a single-crystalline bar-shaped sample with dimensions $8 \times 2 \times 2 \mathrm{~mm} \mathrm{[3]} \mathrm{was}$ cut perpendicular to the cleavage plane, that is, along the (110) direction. Because of the brittleness of the crystal due to its strong tendency to cleave parallel to the $c$ axis, it was not possible to obtain a sample long enough for low-temperature measurements along the $c$ axis. Electrical resistivity, thermopower, and thermal conductivity were measured simultaneously using the thermal transport option of a physical property measurement system (PPMS, Quantum Design) between 300 and $5 \mathrm{~K}$. The electrical and thermal contacts were made by soldering four copper bars with a low-melting-point braze. The experimental uncertainty in the measurements of these three transport coefficients is estimated to be $5 \%$ for each. Specific-heat measurement was performed between 2 and $300 \mathrm{~K}$ under zero magnetic field using the 
${ }^{4} \mathrm{He}$ specific-heat option of the PPMS. For this measurement, a small cleaved piece of approximately $20 \mathrm{mg}$ was glued onto the sample holder using a minute amount of Apiezon $\mathrm{N}$ grease. Additional measurements between 2 and $0.35 \mathrm{~K}$ were carried out using the ${ }^{3} \mathrm{He}$ specific-heat option of the PPMS.

Inelastic neutron scattering (INS) measurements were performed on the triple-axis spectrometer 1T at the Laboratoire Léon Brillouin (LLB) in Saclay (France). All measurements were carried out with a final neutron wave vector of $k_{f}=$ $2.662 \AA^{-1}\left(E_{f}=14.7 \mathrm{meV}\right)$. A focusing pyrolytic graphite (PG) (002) monochromator and analyzer were used, with a PG filter inserted into the scattered beam in order to eliminate higher-order contamination. A small piece of singlecrystalline InTe was mounted on the cold finger of a 4-K-Janis closed-cycle cryostat. The sample size $\left(0.5 \times 0.5 \mathrm{~mm}^{2}\right.$ and $1.5 \mathrm{~mm}$ for in- and out-of-the scattering plane, respectively) was defined to optimize the neutron transmission for energy transfers of few $\mathrm{meV}$ and by considering the strong absorption cross section of elemental In. The sample was aligned in the scattering plane $[100,010]$ such that wave vectors of the form $Q=\frac{2 \pi}{a}(h, k, 0)$ (with a lattice parameter $a=8.4380 \AA$ ) were accessible. Elastic scans through the main intense Bragg peaks in the scattering plane are reported in Appendix F. The good crystalline quality of the single crystal is highlighted by its mosaicity found to be below the instrumental resolution, that is, below $0.1^{\circ}$.

Low-frequency Raman spectroscopy experiments were carried out at room temperature using a LabRAMHR spectrometer (HORIBA Jobin Yvon) operating with an excitation laser line at $532 \mathrm{~nm}$. The signal was collected in backscattering geometry with a long-working distance objective $(50 \times$ magnification). The scattered light was dispersed by 1800 grooves $/ \mathrm{mm}$ and inelastic signal can be detected down to about $8 \mathrm{~cm}^{-1}$.

DFT calculations were conducted using the projector augmented wave (PAW) method, implemented in the Vienna ab initio Simulation Package (VASP) [80]. Several exchange correlation functionals (LDA, GGA-PBE, PBE-sol) have been tested to estimate correctly atomic forces in order to remove imaginary frequencies in the relaxed cell. Since none of them have been able to, we have chosen to only present results described by the generalized gradient approximation modified by Perdew, Burke, and Ernzerhof (GGA-PBE) [81]. A planewave basis set with a cutoff energy $E=600 \mathrm{eV}$ was used in all calculations. A high-density $k$-point meshing was employed for Brillouin-zone integrations $(15 \times 15 \times 17)$. These parameters ensured good convergence for the total energy. The convergence tolerance for the calculations was selected as a difference of the total energy within $10^{-6} \mathrm{eV}$. Upon performing volume and ionic relaxation steps, Blöchl correction was applied in a final step calculation [82]. Phonon-dispersion bands, phonon density of states (PDOS), and vibrational free energy at $T>0 \mathrm{~K}$ were obtained by computing the atomic forces for different, finite atomic displacements, and a subsequent calculation and integration over the corresponding phonon frequencies, within the harmonic approximation, i.e., without any volume dependence, using the PHONOPY code [83]. The phonon calculations were carried out within a $3 \times 3$ $\times 3$ of the primitive cell, i.e., using a supercell of 216 atoms with five atomic nonequivalent displacements. Specific-heat calculations within the quasiharmonic approximation were realized by computing the free energy $G(T, p)$ as a function of volume $V$, from a sequence of harmonic phonon calculations, and by calculating an equation of state fitted at each temperature. More details about this method can be found in Ref. [84]. The specific heat $C_{p}$ was then computed from the equation $C_{p}(T, p)=-T \frac{\partial^{2} G(T, p)}{\partial T^{2}}$.

\section{APPENDIX B: CRYSTALLOGRAPHIC DATA COLLECTED AT 300 AND $80 \mathrm{~K}$}

The main crystallographic parameters of InTe obtained at 300 and $80 \mathrm{~K}$ and details of the structural refinements are given in Table I. The atomic coordinates and isotropic thermal

TABLE I. Crystal data and structure refinements of single-crystalline InTe at 300 and $80 \mathrm{~K}$.

\begin{tabular}{lcc}
\hline \hline Temperature $(\mathrm{K})$ & $300 \mathrm{~K}$ & $80 \mathrm{~K}$ \\
Molar mass $\left(\mathrm{g} \mathrm{mol}^{-1}\right)$ & 242.42 & 242.42 \\
Symmetry & tetragonal & tetragonal \\
Space group & $I 4 / m c m$ & $I 4 / m c m$ \\
$a(\AA)$ & $8.438(1)$ & $8.345(3)$ \\
$c(\AA)$ & $7.141(1)$ & $7.107(3)$ \\
$V\left(\AA^{3}\right)$ & $508.46(2)$ & $498.50(2)$ \\
$Z$ & 8 & 8 \\
$\rho_{\text {calc }}\left(\mathrm{g} \mathrm{cm}^{-3}\right)$ & 6.33 & 6.46 \\
$2 \theta$ range $\left(^{\circ}\right)$ & $1.60-98.23$ & $1.64-72.89$ \\
Radiation $(\AA)$ & Mo- $K \alpha ; \lambda=0.71073$ & ( $)$ \\
Absorption coefficient $\left(\mathrm{mm}^{-1}\right)$ & 20.12 & 20.53 \\
Index range & $-15 \leqslant h \leqslant 17$ & $-11 \leqslant h \leqslant 14$ \\
& $-17 \leqslant k \leqslant 17$ & $-12 \leqslant k \leqslant 12$ \\
& $-15 \leqslant l \leqslant 15$ & $-11 \leqslant l \leqslant 11$ \\
Data/restraints/parameters & $9274 / 0 / 717$ & $4486 / 0 / 349$ \\
$R$ indices $\left[F_{\mathrm{o}}>4 \sigma\left(F_{\mathrm{o}}\right)\right]$ & $R 1=0.0255$ & $R 1=0.0542$ \\
& $w R 2=0.0431$ & $w R 2=0.1082$ \\
Goodness of fit on $F^{2}$ & 2.041 & 2.436 \\
\hline \hline
\end{tabular}


TABLE II. Atomic coordinates and equivalent isotropic displacement parameters $\left(\AA^{2}\right)$ for single-crystalline InTe at $300 \mathrm{~K} . U_{\text {eq }}$ is defined as one-third of the trace of the orthogonalized $U_{\mathrm{ij}}$ tensor.

\begin{tabular}{lcccc}
\hline \hline Atom & $x$ & $y$ & $z$ & $U_{\text {eq }}$ \\
\hline In1 & 0 & 0 & 0.25 & $0.0539(2)$ \\
In2 & 0 & 0.5 & 0.25 & $0.0177(1)$ \\
Te & $0.18223(2)$ & $-0.31777(2)$ & 0 & $0.0167(1)$ \\
\hline \hline
\end{tabular}

displacement parameters as well as the anisotropic thermal displacement parameters inferred at $300 \mathrm{~K}$ are listed in Tables II and III, respectively. The same parameters determined at $80 \mathrm{~K}$ are gathered in Tables IV and V, respectively.

\section{APPENDIX C: CALCULATION OF THE SPECIFIC HEAT WITHIN THE QUASIHARMONIC APPROXIMATION}

As explained in the main text, phonon calculations using the experimental parameters [model (i)] and using the DFT relaxed cells [model (ii)] exhibit imaginary frequencies. Thus, we have chosen only model (iii) (by compressing the unit-cell volume to $88 \%$ ) to estimate the specific heat [Fig. 8(a)]. However, the estimation of $C_{p}$ within the quasiharmonic approximation requires to perform phonon calculations within the harmonic approximation using different volumes (including $c / a$ ratio and internal parameters optimization for each volume) and to minimize the free energy as a function of temperature and volume. Since a highly pressurized cell ( $13 \mathrm{GPa}, V=0.73 V_{0}$ where $V_{0}$ is the initial volume) is still mechanically stable, the extrapolation of the curvature of the Helmholtz energies, obtained only from mechanically stable calculations, was shifted towards a volume of $0.88 V_{0}$ to estimate $C_{p}$ [Fig. 8(b)]. Considering this approximation, the results should be taken with some care for high temperatures but are nevertheless reasonable below room temperature.

\section{APPENDIX D: SPECIFIC HEAT, ELECTRICAL RESISTIVITY, AND TOTAL THERMAL CONDUCTIVITY}

The temperature dependences of the specific heat, electrical resistivity and total thermal conductivity for singlecrystalline InTe are shown in Figs. 9(a), 9(b) and 9(c), respectively.

TABLE III. Anisotropic thermal displacement parameters $\left(\AA^{2}\right)$ for single-crystalline InTe at $300 \mathrm{~K}$. By symmetry, $U_{23}, U_{12}$, and $U_{13}$ are equal to zero for In 1 and In 2 atoms.

\begin{tabular}{lcccc}
\hline \hline Atom & $U_{11}$ & $U_{22}$ & $U_{33}$ & $U_{12}$ \\
\hline In1 & $0.0424(3)$ & $0.0424(5)$ & $0.0770(6)$ & 0 \\
In2 & $0.0214(1)$ & $0.0214(1)$ & $0.0103(1)$ & 0 \\
Te & $0.0172(1)$ & $0.0172(1)$ & $0.0156(1)$ & $-0.0025(1)$ \\
\hline \hline
\end{tabular}

TABLE IV. Atomic coordinates and equivalent isotropic displacement parameters $\left(\AA^{2}\right)$ for single-crystalline InTe at $80 \mathrm{~K} . U_{\text {eq }}$ is defined as one-third of the trace of the orthogonalized $U_{\mathrm{ij}}$ tensor.

\begin{tabular}{lcccc}
\hline \hline Atom & $x$ & $y$ & $z$ & $U_{\text {eq }}$ \\
\hline In1 & 0 & 0 & 0.25 & $0.0245(5)$ \\
In2 & 0 & 0.5 & 0.25 & $0.0071(4)$ \\
Te & $0.18351(9)$ & $-0.31649(9)$ & 0 & $0.0070(3)$ \\
\hline \hline
\end{tabular}

\section{APPENDIX E: BRILLOUIN ZONE AND ELECTRONIC BAND STRUCTURE OF InTe}

The electronic dispersion curves along high symmetry directions, shown in Figs. 10(a) and 10(b), have been calculated using a compressed unit cell and the experimental lattice parameters, respectively.

\section{APPENDIX F: ADDITIONAL INELASTIC NEUTRON-SCATTERING DATA}

Transverse-acoustic phonons have been measured around the Bragg peaks (600) and (330). Elastic scans through these Bragg peaks performed along transverse directions in the reciprocal space, i.e., along the [010] and [-110] directions, respectively, are shown in Fig. 11. In this geometry, the observed widths of the Bragg peaks are mostly determined by the instrumental resolution and the crystal mosaicity. The fits of both intensity profiles give an intrinsic crystal mosaicity of $0.23^{\circ}$.

Figure 12 shows the energy scans taken on the three-axis spectrometer1T@LLB around the strong Bragg peak (600) along the [010] direction so that it corresponds to transverseacoustic phonons propagating along the [010] direction with the polarization along [100]. Figure 13 shows the energy scans around the strong Bragg peak (330) along the $[-110]$ direction, corresponding to transverse-acoustic phonons propagating along the $[-110]$ direction polarized along [110]. The procedure used to extract properly the intrinsic phonon energy width, energy position, and dynamical structure factor, or normalized from the convolution with the four-dimensionnal INS instrumental resolution, has been reported in our previous studies (Refs. [57,78]). The black solid lines in Fig. 12 are fits, which include the convolution between a model cross section (here, the damped harmonic oscillator for the acoustic-phonon mode and a Dirac contribution for the incoherent intensity) with the spectrometer resolution. It allows us to take into ac-

TABLE V. Anisotropic thermal displacement parameters $\left(\AA^{2}\right)$ for single-crystalline InTe at $80 \mathrm{~K}$. By symmetry, $U_{23}, U_{12}$, and $U_{13}$ are equal to zero for In1 and In2 atoms.

\begin{tabular}{lcccc}
\hline \hline Atom & $U_{11}$ & $U_{22}$ & $U_{33}$ & $U_{12}$ \\
\hline In1 & $0.0194(8)$ & $0.0194(8)$ & $0.035(1)$ & 0 \\
In2 & $0.0097(6)$ & $0.0097(6)$ & $0.020(5)$ & 0 \\
Te & $0.0089(4)$ & $0.0089(4)$ & $0.003(3)$ & $-0.0010(4)$ \\
\hline \hline
\end{tabular}




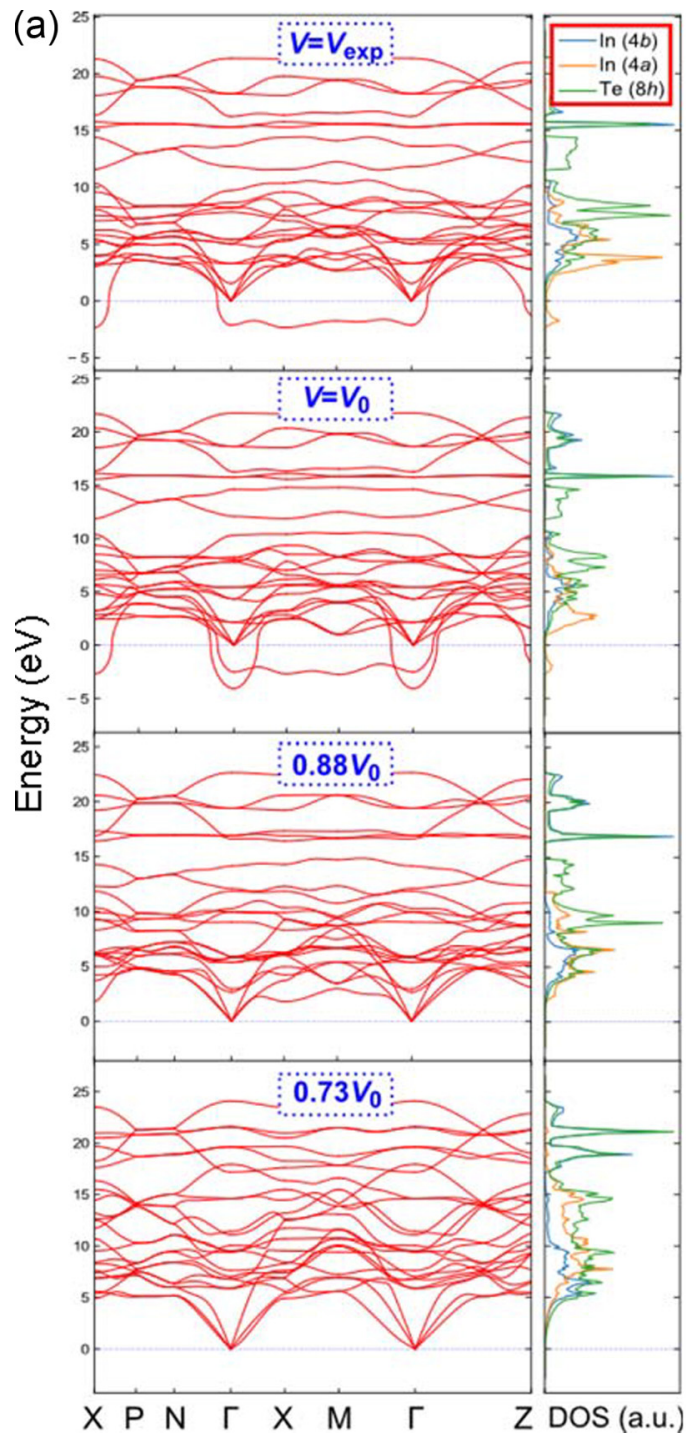

(b)

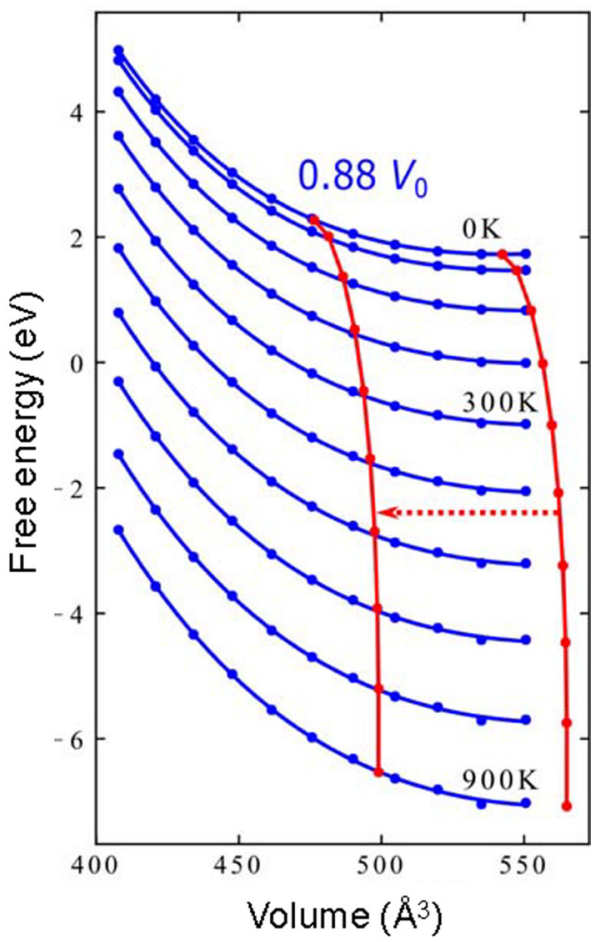

FIG. 8. (a) Phonon-dispersion curves of InTe calculated for several pressurized unit cells: models (i) from experimental parameters, (ii) from DFT relaxed structure, (iii) under $3 \mathrm{GPa}$, and (iv) under $13 \mathrm{GPa}$. (b) Isothermal free-energy curves as a function of volume (blue dots and curves) and behavior of the free-energy minimum as a function of volume (red dots and curves). The curves calculated for the volume of $0.73 V_{0}$ have been shifted towards a volume of $0.88 V_{0}$ used to calculate the phonon-dispersion curves and phonon density of states shown in the main text.

count the local curvature of the phonon branch over the range of the instrumental resolution, i.e., the fact that the energy resolution for an acoustic-phonon mode is not independent of its slope. The blue dotted lines show the contribution of the acoustic phonon only. Of note, only this method allows for a reliable extraction of the characteristics of phonons from INS data. Considering this convolution, variations in the observed width of the acoustic phonons as a function of the wave vector are fully reproduced by the convolution effect and the local curvature of the phonon branch so that no additional intrinsic energy width can be resolved. To exemplify the effect of an intrinsic energy width, some of the fits have been repeated by constraining the phonon energy width to $500 \mu \mathrm{eV}$ [red dotted lines in Figs. 12(c) and 12(e) and Fig. 13(b)]. 
(a)

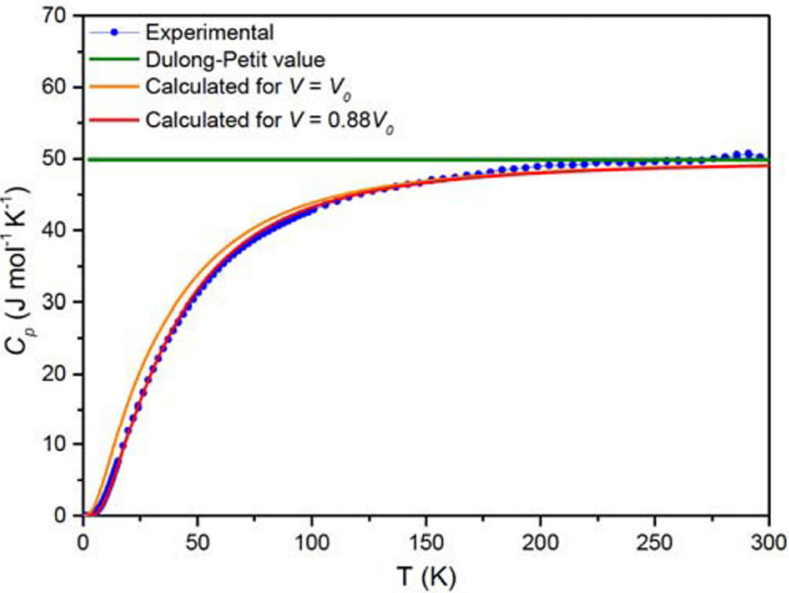

(b)

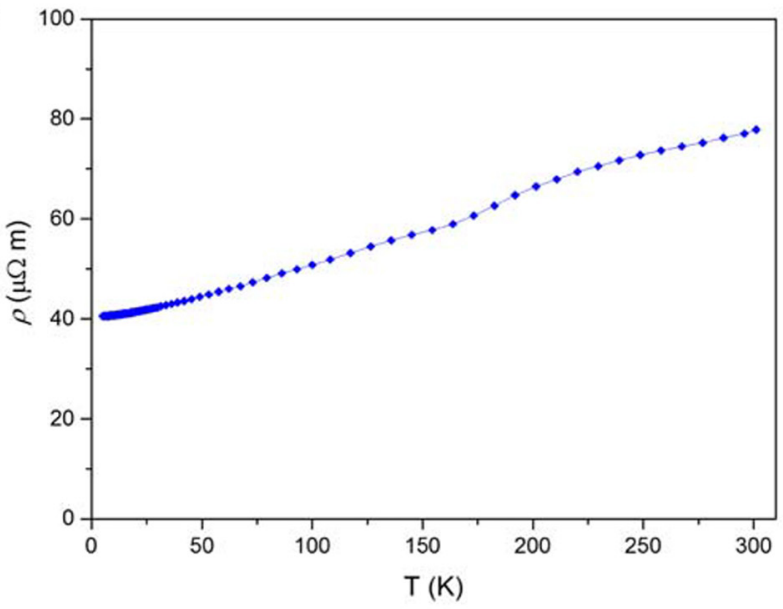

(c)

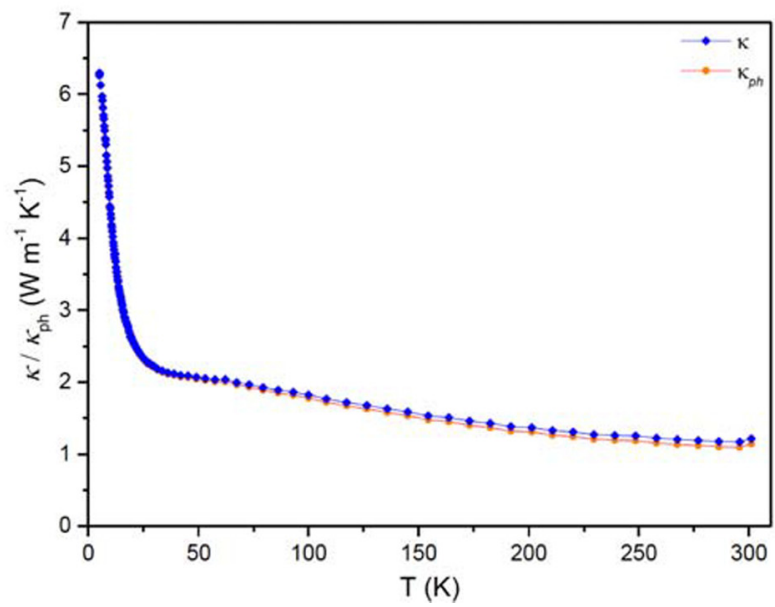

FIG. 9. (a) Comparison of the experimental and theoretical temperature dependencies of the specific heat $C_{p}$ of InTe. The horizontal solid green line represents the Dulong-Petit limit of $3 N R$ where $N$ is the number of atoms per formula unit and $R$ is the ideal gas constant. (b) Temperature dependence of the electrical resistivity $\rho$ measured along the $c$ axis. (c) Comparison of the temperature dependence of the total and lattice thermal conductivities $\kappa$ and $\kappa_{\mathrm{ph}}$, respectively, measured along the $c$ axis. The Lorenz number $L$ used to calculate the electronic contribution $\kappa_{e}=L T / \rho$ has been calculated using a single-parabolic band model.
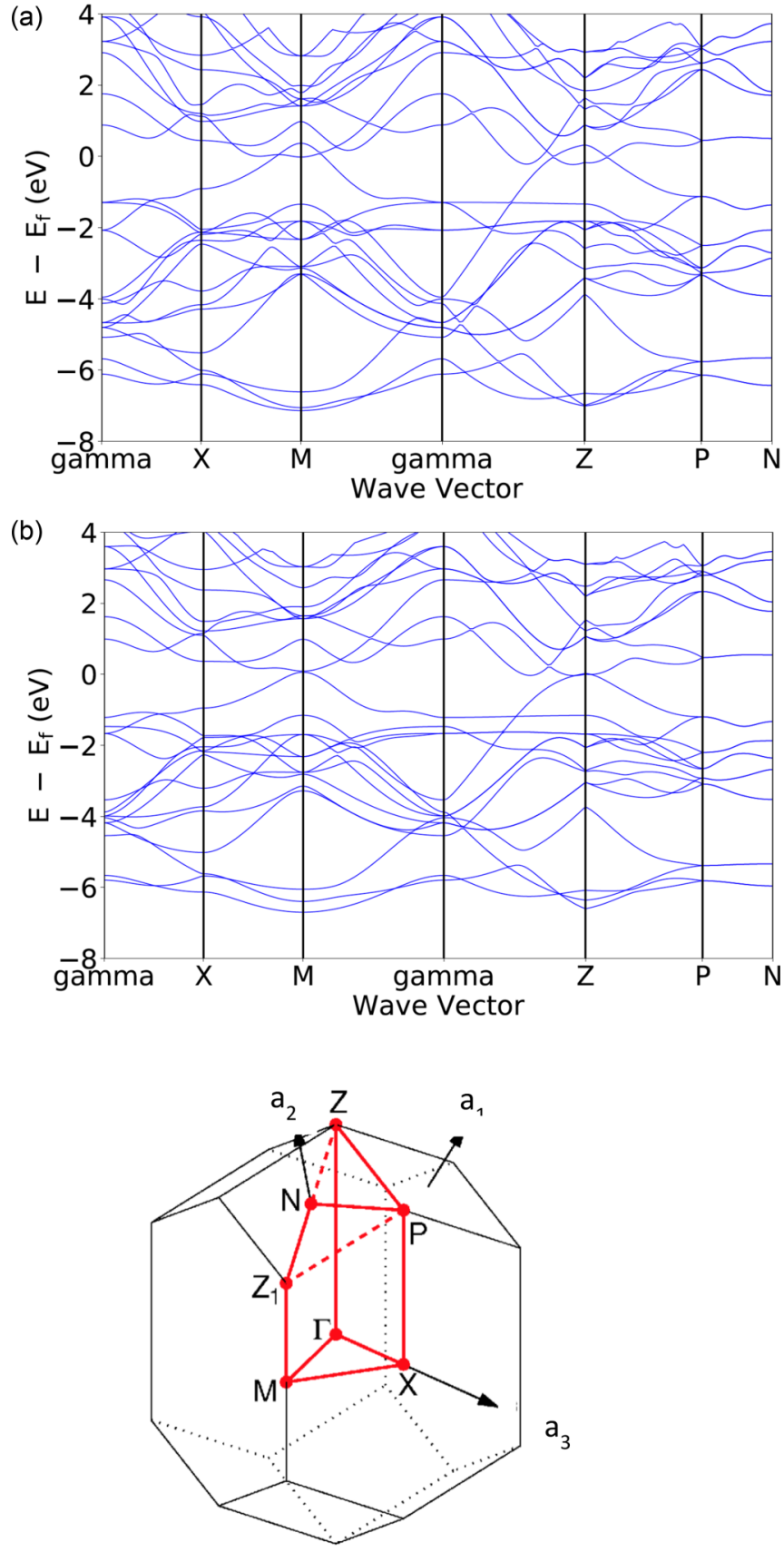

FIG. 10. Electronic band structure of InTe calculated (a) using a unit-cell volume of $0.88 V_{0}$ used in phonon calculations and (b) using the experimental lattice parameters. (c) Brillouin zone of the bodycentered-tetragonal unit cell of InTe. The high-symmetry points are indicated. 


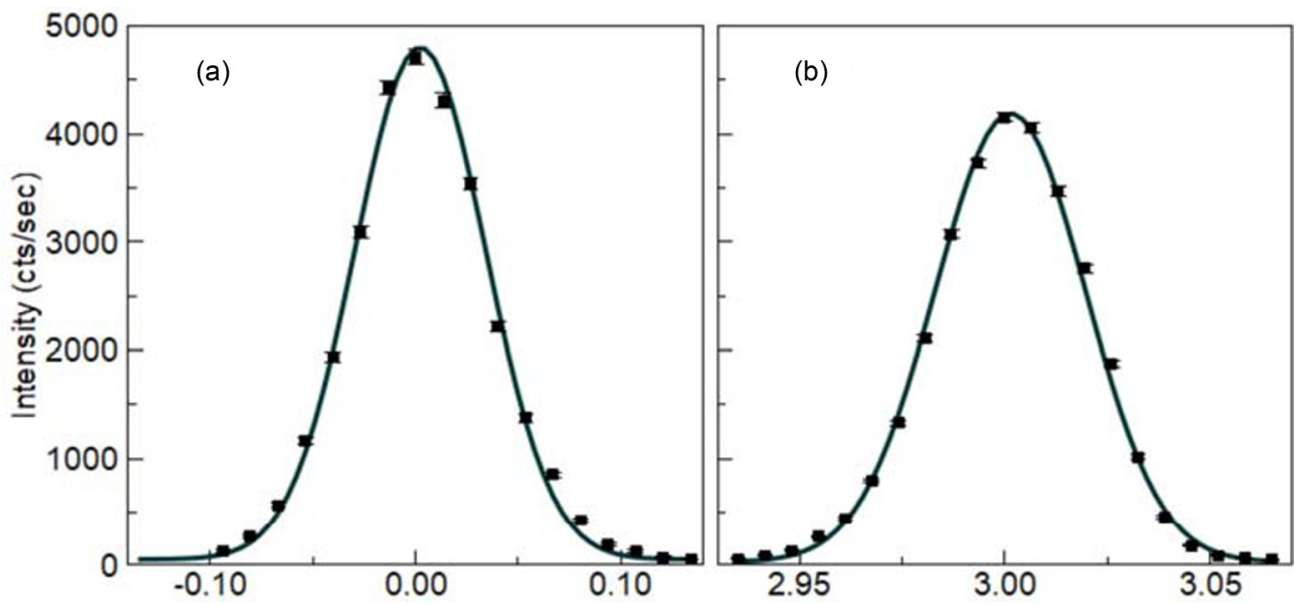

FIG. 11. Elastic scans performed across (a) the (600) and (b) (330) Bragg peaks along the [010] and [-110] directions, respectively. The solid lines stand for the best fit as described in the text.

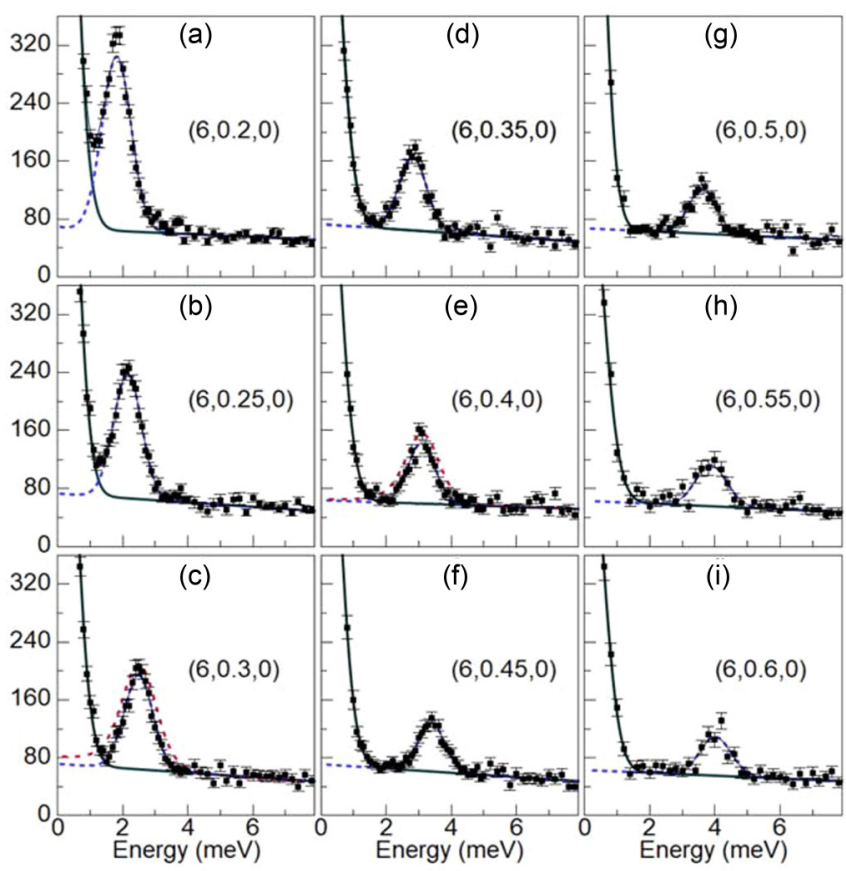

FIG. 12. Energy scans at constant wave vectors across the transverse-acoustic (TA) dispersion measured on the three-axis spectrometer1T@LLB around the Bragg peak (600). The total transferred wave vectors are indicated in the subfigures, with the phonon wave vector corresponding to $q=Q-(600)$. The solid black lines are fits as explained in the text. The dotted red lines in panels (e) and (c) show the fits for which a finite energy width of $500 \mu \mathrm{eV}$ has been imposed.

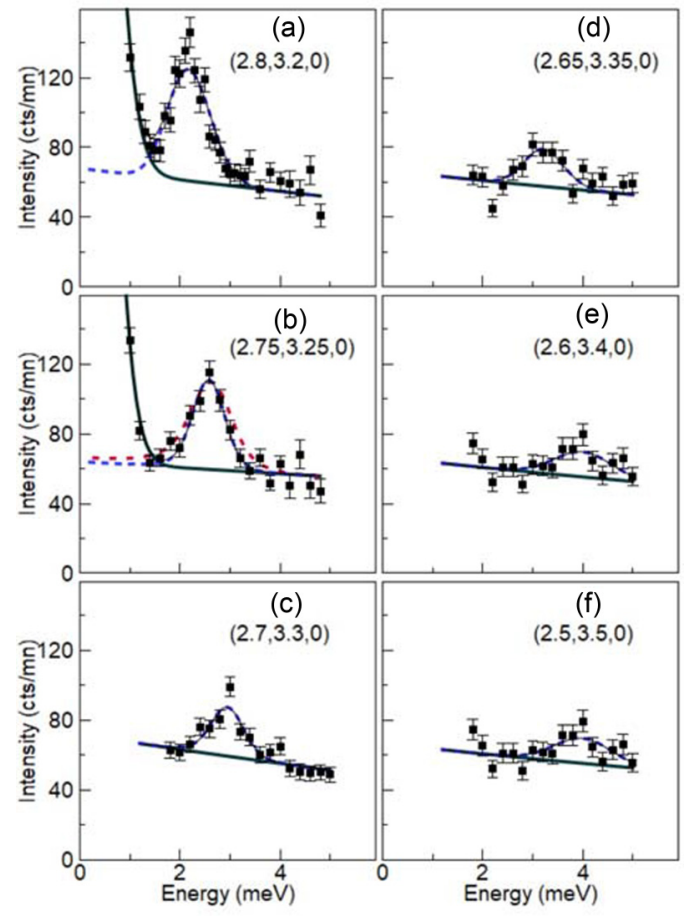

FIG. 13. Energy scans at constant wave vectors across the transverse-acoustic (TA) dispersion measured on the three-axis spectrometer 1T@LLB around the Bragg peak (330). The total transferred wave vectors are indicated in the subfigures, with the phonon wave vector corresponding to $q=Q-$ (330). The solid black lines are fits as explained in the text. The dotted red line in panel (b) shows the fits for which a finite-energy width of $500 \mu \mathrm{eV}$ has been imposed. 
[1] H. J. Goldsmid, Thermoelectric Refrigeration (Springer, New York, 1964).

[2] Thermoelectrics and its Energy Harvesting, edited by D. M. Rowe (CRC, Boca Raton, FL, 2012).

[3] J. Klarbing, O. Hellman, I. A. Abrikosov, and S. I. Simak, Anharmonicity and Ultralow Thermal Conductivity in LeadFree Halide Double Perovskites, Phys. Rev. Lett. 125, 045701 (2020).

[4] E. S. Toberer, L. L. Baranowski, and C. Dames, Advances in thermal conductivity, Annu. Rev. Mater. Res. 42, 179 (2012).

[5] E. S. Toberer, A. Zevalkink, and G. J. Snyder, Phonon engineering through crystal chemistry, J. Mater. Chem. A 21, 15843 (2011).

[6] Y. Yu, M. Cagnoni, O. Cojocaru-Mirédin, and M. Wuttig, Chalcogenide thermoelectrics empowered by an unconventional bonding mechanism, Adv. Funct. Mater. 30, 1904862 (2020).

[7] J. Mao, Z. Liu, J. Zhou, H. Zhu, Q. Zhang, G. Chen, and Z. Ren, Advances in thermoelectrics, Adv. Phys. 67, 69 (2018).

[8] M. Wuttig, V. L. Deringer, X. Gonze, C. Bichara, and J.-Y. Raty, Incipient metals: Functional materials with a unique bonding mechanism, Adv. Mater. 30, 1803777 (2018).

[9] J. Hong and O. Delaire, Phase transition and anharmonicity in SnSe, Mater. Today Phys. 10, 100093 (2019).

[10] C. W. Li, J. Hong, A. F. May, D. Bansal, S. Chi, T. Hong, G. Ehlers, and O. Delaire, Orbitally driven giant phonon anharmonicity in SnSe, Nat. Phys. 11, 1063 (2015).

[11] W. Jantsch, in Dynamical Properties of IV-VI Compounds, Springer Tracts in Modern Physics Vol. 99 (Springer-Verlag, Berlin, 1983), pp. 1-50.

[12] O. Delaire, J. Ma, K. Marty, A. F. May, M. A. McGuire, M.-H. Du, D. J. Singh, A. Podlesnyak, G. Ehlers, M. D. Lumsden, and B. C. Sales, Giant anharmonic phonon scattering in $\mathrm{PbTe}$, Nat. Mater. 10, 614 (2011).

[13] M. K. Jana, K. Pal, U. V. Waghmare, and K. Biswas, The origin of ultralow thermal conductivity in InTe: Lonepair-induced anharmonic rattling, Angew. Chem. 128, 7923 (2016).

[14] S. Y. Back, H. Cho, Y.-K. Kim, S. Byeon, H. Jin, K. Koumoto, and J.-S. Rhyee, Enhancement of thermoelectric properties by lattice softening and energy band gap control in Te-deficient $\operatorname{InTe}_{1-\delta}$, AIP Adv. 8, 115227 (2018).

[15] H. Zhu, B. Zhang, G. Wang, K. Peng, Y. Yan, Q. Zhang, X. Han, G. Wang, X. Lu, and X. Zhou, Promoted high temperature carrier mobility and thermoelectric performance of InTe enabled by altering scattering mechanism, J. Mater. Chem. A 7, 11690 (2019).

[16] S. Y. Back, Y.-K. Kim, H. Cho, M.-K. Han, S.-J. Kim, and J.-S. Rhyee, Temperature-induced Lifshitz transition driven and charge density wave in $\mathrm{InTe}_{1-\delta}$ thermoelectric materials, ACS Appl. Energy Mater. 3, 3628 (2020).

[17] T. Chattopadhyay, R. P. Santandrea, and H. G. von Schnering, Temperature and pressure dependence of the crystal structure of InTe: a new high pressure phase of InTe, J. Phys. Chem. Solids 46, 351 (1985).

[18] D. W. Liu, C. H. Perry, and A. A. Feinberg, Neutron-scattering studies of phonons in disordered cubic zirconia at elevated temperatures, Phys. Rev. B 36, 9212 (1987).
[19] T. Shirakami, T. Atake, T. Mori, and H. Yamamura, Lowtemprature heat capacity of $\left(\mathrm{ZrO}_{2}\right)_{1-x}\left(\mathrm{Y}_{2} \mathrm{O}_{3}\right)_{x}(x=0.0776$ and 0.0970) and low energy excitations, Solid State Ion 79, 143 (1995).

[20] D. G. Cahill, S. K. Watson, and R. O. Pohl, Lower limit to the thermal conductivity of disordered crystals, Phys. Rev. B 46, 6131 (1992).

[21] N. H. Andersen, K. N. Clausen, J. K. Kjems, and J. Schoonman, A study of the disorder in heavily doped $\mathrm{Ba}_{1-x} \mathrm{La}_{x} \mathrm{~F}_{2+x}$ by neutron scattering, ionic conductivity and specific heat measurements, J. Phys. C 19, 2377 (1986).

[22] K. Suekuni, M. A. Avila, K. Umeo, and T. Takabatake, Cagesize control of guest vibration and thermal conductivity in $\mathrm{Sr}_{8} \mathrm{Ga}_{16} \mathrm{Si}_{30-x} \mathrm{Ge}_{x}$, Phys. Rev. B 75, 195210 (2007).

[23] G. S. Nolas, J. L. Cohn, J. S. Dyck, C. Uher, and J. Yang, Transport properties of polycrystalline type-I Sn clathrates, Phys. Rev. B 65, 165201 (2002).

[24] U. Aydemir, C. Candolfi, H. Borrmann, M. Baitinger, A. Ormeci, W. Carrillo-Cabrera, C. Chubilleau, B. Lenoir, A. Dauscher, N. Oeschler, F. Steglich, and Y. Grin, Crystal structure and transport properties of $\mathrm{Ba}_{8} \mathrm{Ge}_{43} \square_{3}$, Dalton Trans. 39, 1078 (2010).

[25] U. Aydemir, C. Candolfi, A. Ormeci, Y. Oztan, M. Baitinger, N. Oeschler, F. Steglich, and Yu. Grin, Low-temperature thermoelectric, galvanomagnetic, and thermodynamic properties of the type-I clathrate $\mathrm{Ba}_{8} \mathrm{Au}_{x} \mathrm{Si}_{46-x}$, Phys. Rev. B 84, 195137 (2011).

[26] A. Bentien, E. Nishikori, S. Paschen, and B. B. Iversen, Crystal structures, atomic vibration, and disorder of the type-I thermoelectric clathrates $\mathrm{Ba}_{8} \mathrm{Ga}_{16} \mathrm{Si}_{30}, \mathrm{Ba}_{8} \mathrm{Ga}_{16} \mathrm{Ge}_{30}, \mathrm{Ba}_{8} \mathrm{In}_{16} \mathrm{Ge}_{30}$, and $\mathrm{Sr}_{8} \mathrm{Ga}_{16} \mathrm{Ge}_{30}$, Phys. Rev. B 71, 144107 (2005).

[27] U. Aydemir, C. Candolfi, A. Ormeci, M. Baitinger, U. Burkhardt, N. Oeschler, F. Steglich, and Yu. Grin, Electronic band structure and low-temperature transport properties of the type-I clathrate $\mathrm{Ba}_{8} \mathrm{Ni}_{x} \mathrm{Ge}_{46-x-y} \square_{y}$, Dalton Trans. 44, 7524 (2015).

[28] U. Aydemir, C. Candolfi, A. Ormeci, M. Baitinger, N. Oeschler, F. Steglich, and Yu. Grin, High temperature thermoelectric properties of the type-I clathrate $\mathrm{Ba}_{8} \mathrm{Ni}_{x} \mathrm{Ge}_{46-x-y} \square_{y}$, J. Phys.: Condens. Matter 26, 485801 (2014).

[29] C. Candolfi, U. Aydemir, M. M. Koza, M. Baitinger, Yu. Grin, and F. Steglich, Inelastic neutron scattering study of the lattice dynamics in the clathrate compound $\mathrm{BaGe}_{5}$, J. Phys.: Condens. Matter 27, 485401 (2015).

[30] C. Candolfi, M. M. Koza, U. Aydemir, W. Carrillo-Cabrera, Yu. Grin, F. Steglich, and M. Baitinger, Vibrational dynamics of the type-I clathrates $A_{8} \mathrm{Sn}_{44} \square_{2}(A=\mathrm{Cs}, \mathrm{Rb}, \mathrm{K})$ from latticedynamics calculations, inelastic neutron scattering, and specific heat measurements, J. Appl. Phys. 127, 145104 (2020).

[31] D. J. Safarik, T. Klimczuk, A. Llobert, D. D. Byler, J. C. Lashley, J. R. O’Brien, and N. R. Dilley, Localized anharmonic rattling of $\mathrm{Al}$ atoms in $\mathrm{VAl}_{10.1}$, Phys. Rev. B 85, 014103 (2012).

[32] M. M. Koza, A. Leithe-Jasper, E. Sischka, W. Schnelle, H. Borrmann, H. Mutka, and Yu. Grin, Effect of the electropositive elements $A=\mathrm{Sc}, \mathrm{La}$, and $\mathrm{Ce}$ on the microscopic dynamics of $A \mathrm{~V}_{2} \mathrm{Al}_{20}$, Phys. Chem. Chem. Phys. 16, 27119 (2014).

[33] M. M. Koza, H. Mutka, Y. Okamoto, J.-I. Yamaura, and Z. Hiroi, On the microscopic dynamics of the 'Einstein solids' $\mathrm{AlV}_{2} \mathrm{Al}_{20}$ and $\mathrm{GaV}_{2} \mathrm{Al}_{20}$, and of $\mathrm{YV}_{2} \mathrm{Al}_{20}$ : A benchmark system 
for 'rattling' excitations, Phys. Chem. Chem. Phys. 15, 24837 (2015).

[34] K. Suekuni, K. Tsuruta, M. Kunii, H. Nishiate, E. Nishibori, S. Maki, M. Ohta, A. Yamamoto, and M. Koyano, Highperformance thermoelectric mineral CuNiSbS tetrahedrite, J. Appl. Phys. 113, 043712 (2013).

[35] E. Lara-Curzio, A. F. May, O. Delaire, M. A. McGuire, X. Lu, C.-Y. Liu, E. D. Case, and D. T. Morelli, Low-temperature heat capacity and localized vibrational modes in natural and synthetic tetrahedrites, J. Appl. Phys. 115, 193515 (2014).

[36] Y. Bouyrie, C. Candolfi, S. Pailhès, M. M. Koza, B. Malaman, A. Dauscher, J. Tobola, O. Boisron, L. Saviot, and B. Lenoir, From crystal to glass-like thermal conductivity in crystalline minerals, Phys. Chem. Chem. Phys. 17, 19751 (2015).

[37] A. F. May, O. Delaire, J. L. Niedziela, E. Lara-Curzio, M. A. Susner, D. L. Abernathy, M. Kirkham, and M. A. McGuire, Structural phase transition and phonon instability in $\mathrm{Cu}_{12} \mathrm{Sb}_{4} \mathrm{~S}_{13}$, Phys. Rev. B 93, 064104 (2016).

[38] C. Candolfi, G. Guélou, C. Bourgès, A. R. Supka, R. Al Rahal Al Orabi, M. Fornari, B. Malaman, G. Le Caër, P. Lemoine, V. Hardy, J.-M. Zanotti, R. Chetty, M. Ohta, K. Suekuni, and E. Guilmeau, Disorder-driven glasslike thermal conductivity in colusite $\mathrm{Cu}_{26} \mathrm{~V}_{2} \mathrm{Sn}_{6} \mathrm{~S}_{32}$ investigated by Mössbauer spectroscopy and inelastic neutron scattering, Phys. Rev. Mater. 4, 025404 (2020).

[39] R. Viennois, M. M. Koza, R. Debord, P. Toulemonde, H. Mutka, and S. Pailhès, Anisotropic low-energy vibrational modes as an effect of cage geometry in the binary barium silicon clathrate $\mathrm{Ba}_{24} \mathrm{Si}_{100}$, Phys. Rev. B 101, 224302 (2020).

[40] M. Beekman, W. Schnelle, H. Borrmann, M. Baitinger, Yu. Grin, and G. S. Nolas, Intrinsic Electrical and Thermal Properties from Single Crystals of $\mathrm{Na}_{24} \mathrm{Si}_{136}$, Phys. Rev. Lett. 104, 018301 (2010).

[41] A. P. Wilkinson, C. Lind, R. A. Young, S. D. Shastri, P. L. Lee, and G. S. Nolas, Preparation, transport properties, and structure analysis by resonant X-ray scattering of the type-I clathrate $\mathrm{Cs}_{8} \mathrm{Cd}_{4} \mathrm{Sn}_{42}$, Chem. Mater. 14, 1300 (2002).

[42] G. S. Nolas, J.-M. Ward, J. Gryko, L Qiu, and M. A. White, Transport properties of $\mathrm{Na}_{8} \mathrm{Si}_{46}$, Phys. Rev. B 64, 153201 (2001).

[43] B. C. Sales, B. C. Chakoumakos, D. G. Mandrus, and J. W. Sharp, Atomic displacement parameters and the lattice thermal conductivity of clathrate-like thermoelectric compounds, J. Solid State Chem. 146, 528 (1999).

[44] G. S. Nolas, B. C. Chakoumakos, B. Mahieu, G. J. Long, and T. J. R. Weakley, Structural characterization and thermal conductivity of type-I tin clathrates, Chem. Mater. 12, 1947 (2000).

[45] B. C. Sales, D. G. Mandrus, and B. C. Chakoumakos, in Semiconductors and Semimetals (Academic Press, San Diego, 2001), Vol. 70, Chap. 1, pp.1-36.

[46] Z. Hiroi, S. Yonezawa, Y. Nagao, and J. Yamaura, Extremely strong-coupling superconductivity and anomalous lattice properties in the $\beta$-pyrochlore oxide $\mathrm{KOs}_{2} \mathrm{O}_{6}$, Phys. Rev. B 76, 014523 (2007).

[47] M. Christensen, S. Johnsen, and B. B. Iversen, Thermoelectric clathrates of type I, Dalton Trans. 39, 978 (2010).

[48] R. P. Hermann, V. Keppens, P. Bonville, G. S. Nolas, F. Grandjean, G. J. Long, H. M. Christen, B. C. Chakoumakos, B. C. Sales, and D. G. Mandrus, Experimental Evidence for
Atomic Tunneling of Europium in Crystalline $\mathrm{Eu}_{8} \mathrm{Ga}_{16} \mathrm{Ge}_{30}$, Phys. Rev. Lett. 97, 017401 (2006).

[49] R. P. Hermann, W. Schweika, O. Leupold, R. Rüffer, G. S. Nolas, F. Grandjean, and G. J. Long, Neutron and nuclear inelastic neutron scattering study of the Einstein oscillators in Ba-, Sr-, and Eu-filled germanium clathrates, Phys. Rev. B 72, 174301 (2005).

[50] L. Qiu, I. P. Swainson, G. S. Nolas, and M. A. White, Structure, thermal, and transport properties of the clathrates $\mathrm{Sr}_{8} \mathrm{Zn}_{8} \mathrm{Ge}_{38}, \mathrm{Sr}_{8} \mathrm{Ga}_{16} \mathrm{Ge}_{30}$, and $\mathrm{Ba}_{8} \mathrm{Ga}_{16} \mathrm{Si}_{30}$, Phys. Rev. B 70, 035208 (2004).

[51] V. S. Edel'man, Electrons in bismuth, Adv. Phys. 25, 555 (1976).

[52] C. Capillas, E. Kroumova, M. I. Aroyo, J. M. Perez-Mato, H. T. Stokes, and D. M. Hatch, SYMMODES: A software package for group-theoretical analysis of structural phase transitions, J. Appl. Crystallogr. 36, 953 (2003).

[53] M. Nizametdinova, Raman spectrum of InTe and TISe single crystals, Phys. Status Solidi B 97, K9 (1980).

[54] V. Rajaji, K. Pal, S. C. Sarma, B. Joseph, S. C. Peter, U. V. Waghmare, and C. Narayana, Pressure induced band inversion, electronic and structural phase transitions in InTe: A combined experimental and theoretical study, Phys. Rev. B 97, 155158 (2018).

[55] S. Pailhès, H. Euchner, V. M. Giordano, R. Debord, A. Assy, S. Gomès, A. Bosak, D. Machon, S. Paschen, and M. de Boissieu, Localization of Propagative Phonons in a Perfectly Crystalline Solid, Phys. Rev. Lett. 113, 025506 (2014).

[56] H. Euchner, S. Pailhès, V. M. Giordano, and M. de Boissieu, Understanding lattice thermal conductivity in thermoelectric clathrates: A density functional theory study on binary Si-based type-I clathrates, Phys. Rev. B 97, 014304 (2018).

[57] H. Euchner, S. Pailhès, L. T. K. Nguyen, W. Assmus, F. Ritter, A. Haghighirad, Yu. Grin, S. Paschen, and M. de Boissieu, Phononic filter effect of rattling phonons in the thermoelectric clathrate $\mathrm{Ba}_{8} \mathrm{Ge}_{40+x} \mathrm{Ni}_{6-x}$, Phys. Rev. B 86, 224303 (2012).

[58] P.-F. Lory, S. Pailhès, V. M. Giordano, H. Euchner, H. D. Nguyen, R. Ramlau, H. Borrmann, M. Schmidt, M. Baitinger, M. Ikeda, P. Tomes, M. Mihalkovic, C. Allio, M. R. Johnson, H. Schober, Y. Sidis, F. Bourdarot, L. P. Regnault, J. Ollivier, $\mathrm{S}$. Paschen et al. Direct measurement of individual phonon lifetimes in the clathrate compound $\mathrm{Ba}_{7.81} \mathrm{Ge}_{40.67} \mathrm{Au}_{5.33}$, Nat. Commun. 8, 491 (2017).

[59] C. H. Lee, I. Hase, H. Sugawara, H. Yoshizawa, and H. Sato, Low-lying optical phonon modes in the filled skutterudite $\mathrm{CeRu}_{4} \mathrm{Sb}_{12}$, J. Phys. Soc. Jpn. 75, 123602 (2006).

[60] M. K. Jana, K. Pal, A. Warankar, P. Mandal, U. V. Waghmare, and K. Biswas, Intrinsic rattler-induced low thermal conductivity in Zintl type TlInTe 2 , J. Am. Chem. Soc. 139, 4350 (2017).

[61] M. Dutta, S. Matteppanavar, M. V. D. Prasad, J. Pandey, A. Warankar, P. Mandal, A. Soni, U. V. Waghmare, and K. Biswas, Ultralow thermal conductivity in chain-like TlSe due to inherent $\mathrm{Tl}^{+}$rattling, J. Am. Chem. Soc. 141, 20293 (2019).

[62] T. Tadano and S. Tsuneyuki, Quartic Anharmonicity of Rattlers and Its Effect on Lattice Thermal Conductivity of Clathrates from First Principles, Phys. Rev. Lett. 120, 105901 (2018).

[63] Y. Xia, V. Ozolins, and C. Wolverton, Microscopic Mechanisms of Glasslike Lattice Thermal Transport in Cubic $\mathrm{Cu}_{12} \mathrm{Sb}_{4} \mathrm{~S}_{13}$ Tetrahedrites, Phys. Rev. Lett. 125, 085901 (2020). 
[64] D. N. Argyriou and M. M. Elcombe, A neutron scattering investigation of cubic stabilised zirconia (CSZ) - II. Lattice dynamics of Y- and Ca-CSZ, J. Phys. Chem. Solids 57, 343 (1996).

[65] G. P. Cousland, R. A. Mole, M. M. Elcombe, X. Y. Cui, A. E. Smith, C. M. Stampfl, and A. P. J. Stampfl, Investigation of the vibrational properties of cubic yttria-stabilized zirconia: A combined experimental and theoretical study, J. Phys. Chem. Solids 75, 351 (2014).

[66] Q. Xi, Z. Zhang, T. Nakayama, J. Chen, J. Zhou, and B. Li, Off-center rattling triggers high-temperature thermal transport in thermoelectric clathrate: Nonperturbative approach, Phys. Rev. B 97, 224308 (2018).

[67] Y. Liu, Q. Xi, J. Zhou, T. Nakayama, and B. Li, Phonon-glass dynamics in thermoelectric clathrates, Phys. Rev. B 93, 214305 (2016).

[68] Q. Xi, Z. Zhang, J. Chen, J. Zhou, T. Nakayama, and B. Li, Hopping processes explain linear rise in temperature of thermal conductivity in thermoelectric clathrates with off-center guest atoms, Phys. Rev. B 96, 064306 (2017).

[69] P. F. Lory, V. M. Giordano, P. Gille, H. Euchner, M. Mihalkovic, E. Pellegrini, M. Gonzalez, L.-P. Regnault, P. Bastie, H. Schober, S. Pailhès, M. R. Johnson, Yu. Grin, and M. de Boissieu, Impact of structural complexity and disorder on lattice dynamics and thermal conductivity in the $o-\mathrm{Al}_{13} \mathrm{Co}_{4}$ phase, Phys. Rev. B 102, 024303 (2020).

[70] R. Baumbach, F. Bridges, L. Downward, D. Cao, P. Chesler, and B. C. Sales, Off-center phonon scattering sites in $\mathrm{Eu}_{8} \mathrm{Ga}_{16} \mathrm{Ge}_{30}$ and $\mathrm{Sr}_{8} \mathrm{Ga}_{16} \mathrm{Ge}_{30}$, Phys. Rev. B 71, 024202 (2005).

[71] T. Takabatake, K. Suekuni, T. Nakayama, and E. Kaneshita, Rev. Mod. Phys. 86, 669 (2014).

[72] S. O. Long, A. V. Powell, S. Hull, F. Orlandi, C. C. Tang, A. R. Supka, M. Fornari, and P. Vaqueiro, Jahn-Teller driven electronic instability in thermoelectric tetrahedrite, Adv. Funct. Mater. 30, 1909409 (2020).

[73] N. Ghassemi, X. Lu, Y. Tian, E. Conant, Y. Yan, X. Zhou, and J. H. Ross, Structure change and rattling dynamics in
$\mathrm{Cu}_{12} \mathrm{Sb}_{4} \mathrm{~S}_{13}$ tetrahedrite: an NMR study, ACS Appl. Mater. Interfaces 10, 36010 (2018).

[74] T. Nakayama and E. Kaneshita, Significance of Off-Center Rattling for Emerging Low-Lying THz Modes in Type-I Clathrates, J. Phys. Soc. Jpn. 80, 104604 (2011).

[75] R. F. Brebrick and A. J. Strauss, Anomalous thermoelectric power as evidence for two-valence bands in SnTe, Phys. Rev. 131, 104 (1963).

[76] R. F. Brebrick, Deviations from stoichiometry and electrical properties in SnTe, J. Phys. Chem. Solids 24, 27 (1963).

[77] D. Ibrahim, C. Candolfi, S. Migot, J. Ghanbaja, A. Dauscher, G. Le Caër, B. Malaman, C. Semprimoschnig, and B. Lenoir, Comprehensive study of the low-temperature transport properties of polycrystalline $\mathrm{Sn}_{1+x} \mathrm{Te}(x=0$ and 0.03$)$, Phys. Rev. Mater. 3, 085404 (2019).

[78] S. Pailhès, V. M. Giordano, P.-F. Lory, M. de Boissieu, and H. Euchner, X-Rays and Neutrons Spectroscopy for the Investigation of Individual Phonons Properties in Crystalline and Amorphous Solids, in Nanostructured Semiconductors, edited by K. Termentzidis (Jenny Stanford Publishing, New York, 2017), Chap. 19.

[79] L. J. Farrugia, WinGX and ORTEP for Windows: an update, J. Appl. Crystallogr. 45, 849 (2012).

[80] G. Kresse and J. Hafner. Ab initio molecular dynamics for liquid metals, Phys. Rev. B 47, 558 (1993).

[81] J. P. Perdew, K. Burke, and M. Ernzerhof, Generalized Gradient Approximation Made Simple, Phys. Rev. Lett. 77, 3865 (1996).

[82] P. E. Blöchl, O. Jepsen, and O. K. Andersen, Improved tetrahedron method for Brillouin-zone integrations, Phys. Rev. B 49, 16223 (1994).

[83] A. Togo and I. Tanaka, First principles phonon calculations in materials science, Scr. Mater. 108, 1 (2015).

[84] A. Togo, L. Chaput, I. Tanaka, and G. Hug, First-principles phonon calculations of thermal expansion in $\mathrm{Ti}_{3} \mathrm{SiC}_{2}, \mathrm{Ti}_{3} \mathrm{AlC}_{2}$, and $\mathrm{Ti}_{3} \mathrm{GeC}_{2}$, Phys. Rev. B 81, 174301 (2010). 